\title{
METODOLOGIA DE PROJETO DE FILTROS DE SEGUNDA ORDEM PARA INVERSORES DE TENSÃO COM MODULAÇÃO PWM DIGITAL
}

\author{
Leandro Michels* \\ michels@ieee.org \\ Fernando Botterón* \\ botteron@mail.ufsm.br
}

\author{
Robinson F. de Camargo* \\ robinsonfcabol.com.br
}

\author{
Humberto Pinheiro* \\ humbertodctlab.ufsm.br
}

\author{
${ }^{*}$ Universidade Federal de Santa Maria \\ Programa de Pós-Graduação em Engenharia Elétrica \\ Grupo de Eletrônica de Potência e Controle \\ CEP 97105-900, Santa Maria, RS, Brasil, +55 5532208463
}

\begin{abstract}
This paper presents a systematized methodology of secondorder output filter for inverters that synthesize sinusoidal voltage waveforms through digital pulse-width modulation (PWM). The objective of this methodology is to determine the largest corner frequency of the filter that ensure the specification of the maximum total harmonic distortion (THD) admissible in the output voltages of the PWM inverter, for linear and non-linear loads. The proposed methodology is discussed in detail, including the description of the required steps for the derivation of the design procedure for different topologies of voltage inverters and the procedure to obtain the design curve. Finally, it is presented some design examples for singlephase, three-phase three-wire and three-phase four-wire filters topologies. Experimental results have been provided to demonstrate the validity of the proposed design methodology.
\end{abstract}

Artigo Submetido em 01/09/04

1a. Revisão em 06/12/04;

2a. Revisão em 14/03/05;

Aceito sob recomendação do Editor Associado

Prof. Dr. José Antenor Pomilio
KEYWORDS: Voltage-source inverters, digital PWM, filter design procedure.

\section{RESUMO}

Este artigo apresenta uma metodologia sistematizada de projeto de filtros de saída de segunda ordem para inversores que sintetizam tensões senoidais através de modulação por largura de pulso (PWM) digital. O propósito desta metodologia é o de determinar os parâmetros do filtro que assegure a especificação da máxima taxa de distorção harmônica total (THD) admissível nas tensões de saída do inversor PWM, para cargas lineares e não-lineares. A metodologia proposta é discutida em detalhes, incluindo a descrição das etapas necessárias para a derivação do procedimento de projeto para diferentes topologias de inversores de tensão e o procedimento para obtenção das curvas necessárias para o projeto. Finalizando, são apresentados alguns exemplos de projeto para topologias de inversores monofásicos e trifásicos com saída a três fios e a quatro fios. Resultados experimentais são apresentados para demonstrar a validade da metodologia de projeto proposta.

PALAVRAS-CHAVE: Inversores de tensão, PWM digital, procedimento de projeto de filtros. 


\section{INTRODUÇÃO}

Os inversores estáticos de tensão modulados em alta freqüência, com destaque para aqueles com modulação por largura de pulso, têm sido amplamente utilizados para sintetizar tensões senoidais para as mais variadas aplicações, tais como fontes ininterruptas de energia (UPS), geradores de tensões CA de potência (ac power sources) e compensadores de reativos (Mohan et al., 1995). Pelo fato destes inversores apresentarem uma elevada distorção harmônica nas tensões de saída devido às componentes harmônicas de alta freqüência introduzidas pela modulação, é de praxe a introdução de filtros LC passa-baixas entre o inversor e a carga. Para os inversores que operam em freqüências de comutação na ordem de dezenas de $\mathrm{kHz}$, estes filtros são projetados para atender às especificações de projeto da máxima THD aceitável nas tensões de saída, levando em consideração a estratégia de modulação empregada. Já para os inversores que operam em freqüências de comutação superiores a estas, alguns cuidados adicionais devem ser tomados para que os filtros também atendam às especificações com relação aos índices máximos aceitáveis de interferência eletromagnética conduzida (Redl, 1996).

Muitas estratégias diferentes de modulação PWM foram desenvolvidas, principalmente por questões tecnológicas, com o intuito de se obter melhorias de desempenho do inversor e de se obter um espectro harmônico de saída que propiciem uma redução do tamanho dos filtros (Holtz, 1992). As estratégias de modulação podem ser classificadas em dois grupos, de acordo com o tipo de tecnologia eletrônica utilizada para a geração dos sinais de modulação: analógica e digital. Por motivos tecnológicos, as estratégias de modulação geradas analogicamente, tais como o PWM natural e o PWM com injeção de harmônicas (Boost e Ziogas, 1988; Enjeti et al., 1990), foram inicialmente muito estudadas e utilizadas, enquanto as estratégias de modulação geradas digitalmente eram praticamente inexistentes. Em meados da década de 1980 este cenário começou a se alterar devido ao desenvolvimento da eletrônica digital, que tornou as tecnologias digitais de geração de PWM tecnologicamente e economicamente competitivas em relação às analógicas. A partir de então foram desenvolvidas muitas estratégias de modulação PWM digital, dentre as quais se destacam o PWM regularmente amostrado e a modulação space vector (Bowes, 1995; van der Broeck et al., 1988). É importante salientar que mesmo sendo conceitualmente distintas as várias estratégias de modulação PWM digital, é possível se analisar de uma maneira unificada todas as estratégias de modulação PWM digitais obtidas a partir de amostras regularmente espaçadas no tempo através da modulação space-vector, (Bowes e Lai, 1997).
O procedimento de projeto de filtros LC de inversores de tensão para atender às especificações de THD máxima admissível nas tensões de saída, sem entrar no mérito com relação à interferência eletromagnética conduzida de alta freqüência, é constituído de duas etapas distintas. A primeira etapa consiste na determinação da freqüência natural do filtro. Diversas metodologias para determinação da freqüência natural de filtros LC foram apresentadas na literatura (Patel e Hoft, 1973; Dewan e Ziogas, 1979; Ryu et al., 2002). Contudo, foram apresentadas poucas metodologias aplicáveis à modulação PWM digital, tendo sido propostos procedimentos de projeto aplicáveis somente a algumas topologias de inversores específicas (van der Broeck e Miller, 1995; Botterón et al., 2001; Michels et al., 2003). A segunda etapa consiste na obtenção da melhor relação entre as capacitâncias e as indutâncias do filtro para a freqüência natural obtida na etapa anterior. Esta relação deve atender às especificações de projeto, tendo conhecimento dos tipos de carga a serem utilizadas no inversor. Algumas metodologias de projeto foram desenvolvidas para inversores operando com cargas lineares, tais como a que minimiza a energia reativa nos elementos do filtro (Dewan e Ziogas, 1979; Dahono, Purwadi e Qamaruzzaman, 1995) e a que resulta na menor impedância de saída que não ultrapassa um limite máximo de pico de corrente nos interruptores (Botterón et al., 2001). Outras metodologias foram desenvolvidas para os casos em que o inversor opera com cargas não-lineares, com destaque para as mostradas em Vukosavic et al. (1990), Kusko et al. (1990), Kim, Choi e Hong (2000) e Ryu et al. (2002). Nestes casos, é necessário se conhecer os tipos de carga que serão utilizadas assim como a impedância de saída do inversor em malha fechada.

Este trabalho apresenta uma metodologia sistematizada de projeto de filtros de saída de segunda ordem para inversores que sintetizam tensões senoidais através de modulação PWM digital. O objetivo da metodologia é a determinação dos parâmetros do filtro que assegurem a especificação para a THD máxima admissível nas tensões de saída, tanto para cargas lineares quanto não-lineares. Primeiramente, é apresentado um procedimento sistematizado para determinação da freqüência natural de filtros LC de saída, aplicável às diferentes topologias de inversores com modulação por largura de pulso gerada digitalmente. A seguir, é proposta uma nova metodologia para determinação da relação $\mathrm{L} / \mathrm{C}$, válida tanto para cargas lineares quanto não-lineares. Esta metodologia é composta de várias etapas, sendo algumas delas baseadas nos trabalhos descritos anteriormente. O procedimento proposto emprega uma função custo para minimização da energia reativa, impõe limitações para os picos máximos de corrente nos indutores e considera a impedância de saída em malha fechada. Além disso, este procedimento possui algumas características inéditas com relação aos apresentados na literatura, como verificação do 
cumprimento da especificação de sobre-elevação máxima das tensões de saída em degraus de carga e a possibilidade de ser aplicado a diferentes tipos leis de controle. A metodologia proposta é discutida em detalhes, sendo apresentados os passos necessários para a obtenção dos parâmetros do filtro para diversas topologias de inversores, onde se assume que as estratégias de modulação são representadas na forma da modulação space vector. Para ilustrar, são mostradas aplicações da metodologia proposta para algumas topologias de filtros monofásicos e trifásicos a três fios e a quatro fios. $\mathrm{O}$ procedimento proposto é comprovado experimentalmente através de resultados obtidos em um protótipo de $1 \mathrm{kVA}$ para alguns exemplos de projeto.

O artigo está organizado da seguinte forma. A Seção 2 apresenta um resumo de todo o procedimento de projeto proposto, que é descrito em detalhes nas seções subseqüentes. Na Seção 3 é descrita a metodologia para determinação da freqüência natural para inversores monofásicos, cuja análise é sumarizada e estendida para outras topologias. Na Seção 4 é apresentada a metodologia para obtenção da relação entre os parâmetros L e C. A Seção 5 apresenta alguns exemplos de projeto, para os quais foram obtidos resultados experimentais. Finalizando, a Seção 6 apresenta as conclusões do trabalho.

\section{METODOLOGIA DE PROJETO PROPOSTA}

$\mathrm{O}$ procedimento de projeto apresentado baseia-se numa metodologia para a determinação dos parâmetros do filtro que atendam às especificações da THD máxima admissível nas tensões de saída do inversor PWM. Esta metodologia considera, para o projeto, as distorções harmônicas na tensão de saída de duas procedências distintas:

- Distorções harmônicas de alta freqüência, originárias da modulação por largura de pulso. Estas harmônicas são intrínsecas ao inversor, sendo atenuadas através da utilização de filltro LC de saída com freqüência natural suficientemente pequena para satisfatória atenuação;

- Distorções harmônicas de baixa freqüência, originárias das cargas com comportamento não-linear. Estas harmônicas dependem do perfil de corrente drenada pela carga não-linear e da impedância de saída do inversor nestas freqüências, dadas pela relação $\mathrm{L} / \mathrm{C}$ e pela capacidade da lei de controle de atenuá-las (quando o sistema opera em malha fechada).

Experimentalmente, se observa a existência de outras distorções harmônicas de baixa freqüência decorrentes do comportamento não-ideal do inversor. Um exemplo são as distorções harmônias geradas pelos tempos mortos no acionamento dos interruptores. $\mathrm{O}$ procedimento de projeto apresentado desconsidera estas harmônicas.

Os dois tipos de distorções harmônicas considerados na metodologia proposta podem ser analisados independentemente um do outro, uma vez que suas fontes são distintas e as harmônicas de alta-freqüência independem da relação $\mathrm{L} / \mathrm{C}$ e da lei de controle utilizada. Sendo assim, pode-se calcular a THD diretamente como a soma das distorções de alta-freqüência e de baixafreqüência.

Desta forma, o projeto do filtro pode ser, basicamente, dividido nas seguintes etapas:

1. Determinação da porcentagem da THD de saída que será devida às harmônicas de alta e baixa freqüência. Para os casos onde o inversor opera com cargas nãolineares, aconselha-se que a porcentagem da THD de saída devida às harmônicas de alta-freqüência correspondam de $10 \%$ a $20 \%$ do valor total especificado. Já quando o inversor opera somente com cargas lineares, considera-se que toda a THD de saída será originária das componentes harmônicas de alta freqüência;

2. Determinação da freqüência natural do filtro (e conseqüentemente do produto LC) que atenue suficientemente as harmônicas de alta-freqüência, de acordo com o estipulado no passo anterior;

3. Determinação da relação $\mathrm{L} / \mathrm{C}$ e da lei de controle que seja a mais atrativa em relação ao custo dos componentes e que consiga atenuar suficientemente as harmônicas que aparecem devido às cargas nãolineares empregadas para esta aplicação. A THD de saída obtida nesta etapa deve possuir o valor estipulado no passo 1 .

Em muitos casos, pode ser necessário se iterar essas etapas para se atingir a especificação da THD na tensão de saída com cargas não-lineares. Isto irá ocorrer quando as restrições impostas pelo procedimento impedem outra diminuição da relação $\mathrm{L} / \mathrm{C}$, na etapa 3 , para redução da impedância de saída. Neste caso, uma solução é a diminuição da freqüência natural do filtro.

\section{DETERMINAÇÃO DA FREQÜENCIA NATURAL DO FILTRO}

Nesta seção é apresentada, de maneira sistematizada, a derivação do procedimento para a determinação da freqüência natural do filtro. A metodologia apresentada é aplicável em várias topologias de inversores alimentados em tensão, monofásicos e polifásicos, com filtro LC de saída, incluindo inversores multiníveis. 


\subsection{Determinação da freqüência natural do filtro para inversores monofásicos}

Nesta seção será apresentada e discutida a metodologia para determinação da freqüência natural do filtro, de acordo com o valor da THD estipulado na etapa 1 da Seção 2. Para tanto, será tomado como exemplo o inversor monofásico do tipo ponte completa com modulação space vector, mostrado na Fig.1(a), cuja análise pode ser estendida aos demais inversores de tensão com modulação PWM digital. Foram empregadas as seguintes hipóteses sobre o inversor:

1. Os interruptores do inversor são ideais, sendo que os interruptores de um mesmo braço comutam complementarmente e a tensão no barramento $\mathrm{CC}$ é assumida como sendo constante;

2. Considera-se que o espaço das tensões de saída do inversor é dividido em regiões, onde para cada região a seqüência de comutação é definida a priori (Pinheiro et al., 2002). Para o inversor em questão, o espaço das tensões de saída é unidimensional, tal como mostrado na Fig.1(b), existindo duas regiões distintas $S_{1}$ e $S_{2}$, onde as seqüências de comutação são previamente conhecidas.

3. Os contadores utilizados para a geração dos sinais de comando dos interruptores são atualizados em uma freqüência fixa, que é considerada idêntica à freqüência de amostragem do sinal de referência. A relação $m_{s}=f_{s}$ $/ f_{1}$ é definida como a freqüência de amostragem normalizada $\left(m_{s} \in \mathbb{N}^{+}\right)$, onde $f_{s}$ é a freqüência de amostragem e $f_{1}$ é a freqüência do sinal modulado.

A freqüência de amostragem normalizada $\left(m_{s}\right)$ é independente do número de comutações dos interruptores em um período de amostragem. Este é um conceito distinto se comparado com a freqüência de modulação normalizada $\left(m_{f}\right)$ que é freqüentemente empregada em inversores com modulação PWM analógica. Portanto, em inversores com modulação space vector, a freqüência de comutação

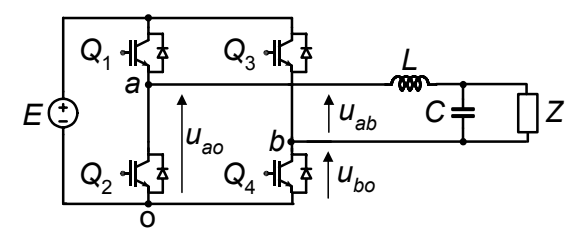

(a)

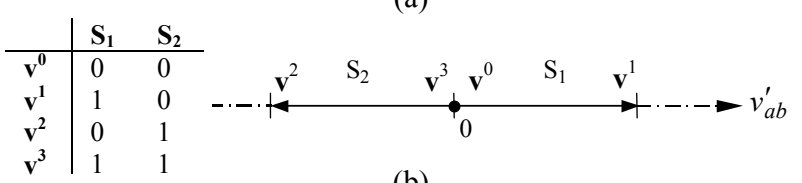

(b)

Figura 1. Inversor monofásico com filtro LC de segunda ordem:

(a) Topologia. (b) Possíveis estados de condução dos interruptores e o espaço das tensões de saída.

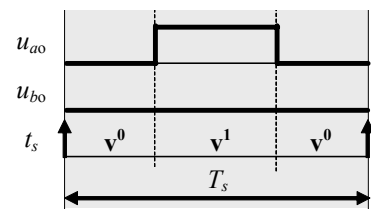

(a)

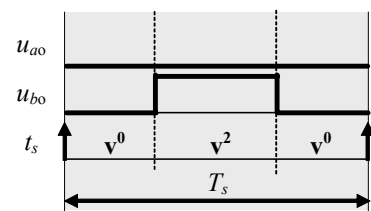

(c)

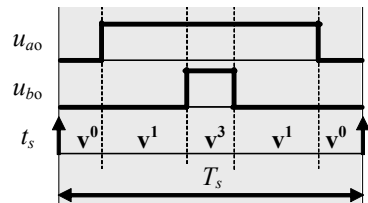

(b)

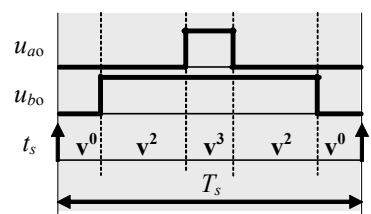

(d)
Figura 2. Sinais de acionamento dos braços do inversor da Fig. 1, em um intervalo de amostragem, com modulação space vector.
(a) Seqüência de comutação 1 , setor $1: S_{1}=\mathbf{v}^{0} \mathbf{v}^{1} \mathbf{v}^{0}$.
(b) Seqüência de comutação 2, setor 1: $S_{1}=\mathbf{v}^{0} \mathbf{v}^{1} \mathbf{v}^{3} \mathbf{v}^{1} \mathbf{v}^{0}$
(c) Seqüência de comutação 1, setor 2: $\mathrm{S}_{2}=\mathbf{v}^{0} \mathbf{v}^{2} \mathbf{v}^{0}$.
(d) Seqüência de comutação 2, setor 2: $\mathrm{S}_{1}=\mathbf{v}^{0} \mathbf{v}^{2} \mathbf{v}^{3} \mathbf{v}^{2} \mathbf{v}^{0}$.

depende da freqüência de amostragem normalizada $m_{s}$ assim como da seqüência de comutação adotada para cada região do espaço das tensões de saída. Para ilustrar esta afirmação, são apresentadas na Fig.2 duas seqüências distintas de comutação para as duas regiões do espaço das tensões de saída do inversor monofásico mostrado na Fig.1. A figura mostra os sinais de acionamento de cada um dos braços do inversor, onde se observa que as seqüências possuem um número diferente de comutações em cada intervalo de amostragem. Realizando a análise espectral de $u_{a b}$ (gerada para um ciclo completo de uma referência senoidal) para as seqüências mostradas na Fig.2, observa-se que os respectivos espectros harmônicos dessas seqüências são distintos, como mostra a Fig.3, apesar de ambas possuírem a mesma freqüência de amostragem.

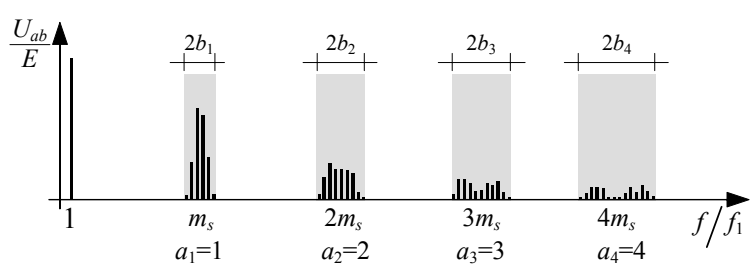

(a)

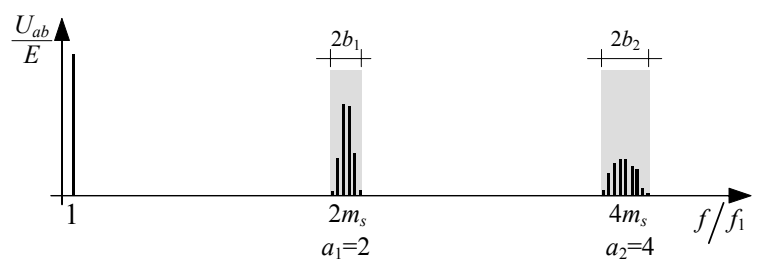

(b)

Figura 3. Espectro de freqüências normalizado de $u_{a b}$ para o inversor da Fig. 1, com modulação space vector, para um sinal modulante senoidal onde $m=0,8$ e $m_{\mathrm{s}}=64$.

(a) Seqüência de comutação $1: S_{1}=\mathbf{v}^{0} \mathbf{v}^{1} \mathbf{v}^{0}, S_{2}=\mathbf{v}^{0} \mathbf{v}^{2} \mathbf{v}^{0}$.

(b) Seqüência de comutação $2: S_{1}=\mathbf{v}^{0} \mathbf{v}^{1} \mathbf{v}^{3} \mathbf{v}^{1} \mathbf{v}^{0}, S_{2}=\mathbf{v}^{0} \mathbf{v}^{2} \mathbf{v}^{3} \mathbf{v}^{2} \mathbf{v}^{0}$. 
A partir das considerações realizadas anteriormente sobre a modulação PWM digital, é obtida a metodologia de projeto para o inversor monofásico de ponte completa mostrado na Fig.1(a). Assumindo-se que o objetivo da metodologia é a determinação da maior freqüência natural do filtro que assegure a especificação de THD nas tensões de saída, toma-se como ponto inicial para obtenção do procedimento de projeto a definição da THD para uma dada tensão:

$$
T H D_{v}=\frac{1}{V_{a b}[1]}\left(\sum_{n=2}^{\infty}\left[V_{a b}[n]\right]^{2}\right)^{1 / 2},
$$

onde $V_{a b}[1]$ e $V_{a b}[n]$ são o valor de pico da componente fundamental e das $n$ harmônicas múltiplas a esta, respectivamente. Note que a tensão $V_{a b}[n]$ pode ser expressa como uma função da tensão $U_{a b}[n]$ de saída do inversor, que é dada por

$$
\left|V_{a b}[n]\right|=|G(s)|_{s=j \omega_{1} n}\left|U_{a b}[n]\right|, \quad n=1,2, \ldots
$$

onde $G(s)$ é a função de transferência entre a saída do inversor e a saída do filtro, que para este filtro é dada por

$$
G(s)=\frac{1}{s^{2} / \omega_{r}{ }^{2}+2 \zeta s / \omega_{r}+1},
$$

onde $\zeta$ é o coeficiente de amortecimento e $\omega_{r}=2 \pi f_{r}$, sendo $\omega_{r}$ e $f_{r}$ a freqüência natural não-amortecida (ou de ressonância) do filtro em $\mathrm{rad} / \mathrm{s}$ e em $\mathrm{Hz}$, respectivamente.

Sendo a função do filtro a de atenuar as altas freqüências geradas pela modulação PWM sem alterar a amplitude da componente fundamental do sinal de referência, pressupõese que a freqüência de ressonância do filtro está localizada entre estes dois grupos de freqüências. Assumindo-se que o espectro de $u_{a b}$ não possui componentes harmônicas entre o sinal modulante de freqüência $f_{1}$ e as harmônicas geradas pela modulação, tal como mostrado na Fig.3, é possível realizar a seguinte aproximação assintótica para (3):

$$
|G(s)| \approx\left|G^{*}(s)\right|=\left\{\begin{array}{c}
1 \quad, s=j \omega_{1} \\
\left(\frac{\omega_{r}}{s}\right)^{2}, s \gg j \omega_{r}
\end{array}\right.
$$

A Fig.4 mostra a resposta em freqüência de $|G(s)|$ e de sua aproximação assintótica $\left|G^{*}(s)\right|$ para diferentes índices de modulação típicos $(\zeta=0$ e $\zeta=1)$. Observa-se nesta figura que a aproximação assintótica possui um erro significativo para as freqüências próximas a $\omega_{r}$, e que este erro é dependente da carga. Por isso, é necessário se investigar em que faixa de freqüências a aproximação por assíntotas apresenta erros pequenos. Para o filtro em questão, observa-se que o erro é inferior a $5 \%$ para $\omega>5 \omega_{r}$ e menor que $1 \%$ para $\omega<0,1 \omega_{r}$, considerando-se $0 \leq \zeta \leq 1$, delimitando, assim, a faixa de freqüências em que a aproximação não é válida. Deve-se

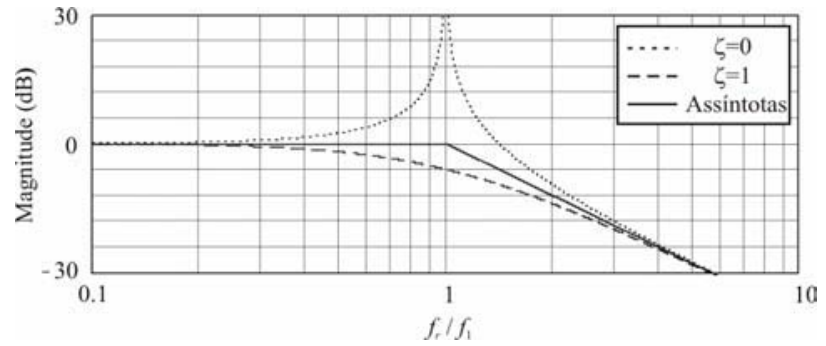

Figura 4. Resposta em freqüência normalizada de $G(s)$, em malha aberta, para $\zeta=0, \zeta=1$ e da sua aproximação por assíntotas.

ressaltar que esta análise está considerando a resposta do inversor em malha aberta. Caso o inversor opere em malha fechada, a restrição quanto ao afastamento espectral entre a freqüência do sinal de referência e a freqüência natural do filtro fica sendo dada pela resposta em freqüência do sistema em malha fechada.

Assim, assumindo que $\omega_{1} \leq 0,1 \omega_{r}$ e que a modulação PWM não gera harmônicas de baixa ordem na faixa entre $0,1 \omega_{r} \leq \omega \leq 5 \omega_{r}$, assegura-se que a aproximação assintótica dada em (4) descreve adequadamente o espectro de harmônicas na saída do filtro. Sabendo-se que só existem harmônicas múltiplas da freqüência fundamental, pode se escrever $f$ como sendo:

$$
f=\frac{\omega_{1} n}{2 \pi}=n f_{1}, n \in \mathbb{N}^{+} .
$$

Desta forma, o espectro da tensão de saída pode ser reescrito como sendo:

$$
\left|V_{a b}[n]\right| \approx\left\{\begin{array}{cl}
\mid U_{a b}[1] \quad, n=1 \\
\left(\frac{f_{r}}{f_{1}}\right)^{2} \frac{1}{n^{2}}\left|U_{a b}[n]\right|, & \forall n \in \mathbb{N}^{+} \mid n \gg \frac{f_{r}}{f_{1}}
\end{array}\right.
$$

onde $f_{r} / f_{1}=\omega_{r} / \omega_{1}$.

Substituindo-se (6) na expressão da $T H D_{v}$ dada em (1), obtém-se:

$$
T H D_{v}=\frac{1}{\left|U_{a b}[1]\right|}\left(\sum_{n=f_{r} / f_{1}}^{\infty}\left[\left(\frac{f_{r}}{f_{1}}\right)^{2} \frac{1}{n^{2}} \mid U_{a b}[n]\right]^{2}\right)^{1 / 2} .
$$

Sabendo que as componentes harmônicas de $u_{a b}$ com amplitude significativa estão localizadas próximas as freqüências múltiplas e submúltiplas da freqüência de amostragem, tal como mostrado na Fig. 3, pode-se reescrever (7) da seguinte forma: 


$$
\begin{aligned}
T H D_{v}=\frac{1}{\left|U_{a b}[1]\right|} & \left(\sum_{n=a_{1} m_{s}-b_{1}}^{a_{1} m_{s}+b_{1}}\left[\left(\frac{f_{r}}{f_{1}}\right)^{2} \frac{1}{n^{2}} \mid U_{a b}[n]\right]^{2}+\right. \\
& \left.+\sum_{n=a_{2} m_{s}-b_{2}}^{a_{2} m_{s}+b_{2}}\left[\left(\frac{f_{r}}{f_{1}}\right)^{2} \frac{1}{n^{2}} \mid U_{a b}[n]\right]^{2}+\cdots\right)^{1 / 2},
\end{aligned}
$$

onde $a_{1}, a_{2}, \ldots$ são as posições espectrais relativas dos grupos de harmônicas de alta ordem em relação à freqüência de amostragem $m_{s}$ do espectro de $u_{a b}$. Por exemplo, para a topologia de inversor apresentada, para a seqüência de comutação $\mathrm{S}_{1}, a_{1}=1, a_{2}=2, \ldots$ e para a seqüência $\mathrm{S}_{2}, a_{1}=2, a_{2}=4, \ldots$. Os índices $b_{1}, b_{2}, \ldots$ delimitam a largura das bandas laterais ao redor de $a_{1} m_{s}, a_{2} m_{s}, \ldots$, respectivamente, onde as componentes harmônicas de $u_{a b}$ tem amplitude significativa, por exemplo, maior que $0,1 \%$ $V_{a b}[1]$. A Fig. 3 ilustra estas aproximações.eguinte forma:

Assumindo que $a_{1} m_{s}, a_{2} m_{s}, \ldots$ são muito maiores que $b_{1}$, $b_{2}, \ldots, \quad\left(b_{1}<0,1 a_{1} m_{s}, \quad b_{2}<0,1 a_{2} m_{s}, \ldots\right) \quad$ e sabendo que as amplitudes das harmônicas são espelhadas em torno das freqüências múltiplas a de comutação $\left(U_{a b}\left[a_{1} m_{s^{-}}\right.\right.$ $\left.\left.b_{1}\right]=U_{a b}\left[a_{1} m_{s}+b_{1}\right], \ldots\right)$, a seguinte aproximação é válida:

$$
\begin{aligned}
& a_{1} m_{s}-b_{1} \approx a_{1} m_{s}+b_{1} \approx a_{1} m_{s}, \\
& a_{2} m_{s}-b_{2} \approx a_{2} m_{s}+b_{2} \approx a_{2} m_{s}, \cdots
\end{aligned}
$$

Assim, a eq. (8) pode ser simplificada como segue:

$$
T H D_{v}=\left(\frac{f_{r}}{f_{1}}\right)^{2} \frac{1}{m_{s}^{2}} n D F_{2}(m),
$$

onde

$$
\begin{aligned}
n D F_{2}(m)=\frac{1}{\left|U_{a b}[1]\right|}\left(\frac{1}{a_{1}^{4}} \sum_{n=a_{1} m_{s}-b_{1}}^{a_{1} m_{s}+b_{1}}\left|U_{a b}[n]\right|^{2}+\right. \\
\left.+\frac{1}{a_{2}^{4}} \sum_{n=a_{2} m_{s}-b_{2}}^{a_{2} m_{s}+b_{2}}\left|U_{a b}[n]\right|^{2}+\cdots\right)^{1 / 2,}
\end{aligned}
$$

sendo $m=U_{a b}[1] / E$ o índice de modulação do sinal modulante. Observa-se que o fator $n D F_{2}(m)$ é uma função não-linear, normalizada, do espectro dos sinais gerados pelo inversor para cada índice de modulação. Para evitar a necessidade de cálculo deste fator em cada projeto, este fator é usualmente traçado na forma de uma curva de projeto, conforme será mostrado a seguir.

Reescrevendo-se (11), chega-se a equação empregada para a determinação da freqüência natural do filtro, dada por:

$$
f_{r}=f_{1} m_{s} \sqrt{\frac{T H D_{v}}{n D F_{2}(m)}}
$$

Equações idênticas a (12) podem ser obtidas para outras topologias de inversores empregando o procedimento apresentado nesta seção. Entretanto, esta extensão não é direta para todos os outros inversores, principalmente para aqueles com múltiplas saídas. Por esse motivo, a seção que segue apresenta os passos que devem ser seguidos para garantir a extensão deste procedimento a outras topologias de inversores e filtros.

\subsection{Determinação da freqüência natural do filtro para outras topologias de inversores}

A extensão do procedimento para a determinação da freqüência natural do filtro para outras topologias de inversores emprega a mesma equação dada em (12), onde o fator $n D F_{2}(m)$ dependerá da função de transferência do mesmo e do padrão PWM injetado no mesmo. Esta seção apresenta as etapas necessárias para a obtenção do fator $n D F_{2}(m)$ para cada topologia de inversor, partindo inicialmente do conjunto de hipóteses que precisam ser satisfeitas para se garantir a validade do procedimento proposto.

\subsubsection{Hipóteses a serem satisfeitas}

Com relação aos sinais de referência, assume-se que:

1. Os sinais de referência são senoidais. No caso de inversores polifásicos, todas as referências devem possuir a mesma freqüência. Além disso, é necessário assegurar que é possível a síntese destes sinais no espaço das tensões de saída do inversor.

2. A partir dos sinais de referência são calculados os sinais de modulação, que são os sinais que efetivamente são empregados para a geração dos sinais de comando dos interruptores. Os sinais de modulação devem ser calculados de tal forma a se garantir a geração das formas de onda desejadas na saída do filtro. Por exemplo, se os inversores forem projetados para operarem de forma equilibrada, tais como os inversores trifásicos a três fios, considera-se que os sinais de modulação são tais que se permite obter formas de onda com mesma freqüência e amplitude na saídas defasadas entre si em $120^{\circ}$. Em alguns casos, isso pode exigir que estes sinais possuam amplitudes diferentes, tal como ocorre no inversor trifásico a três fios com um braço capacitivo (Michels et al., 2003).

3. Os sinais de modulação são sintetizados pelo inversor através de uma técnica de modulação por largura de pulso. Os tempos de condução dos interruptores são calculados a partir de amostras regularmente espaçadas no tempo dos sinais de modulação, sendo o intervalo entre amostras um múltiplo inteiro do período dos sinais de referência, tal como ilustrado na Fig. 5. 


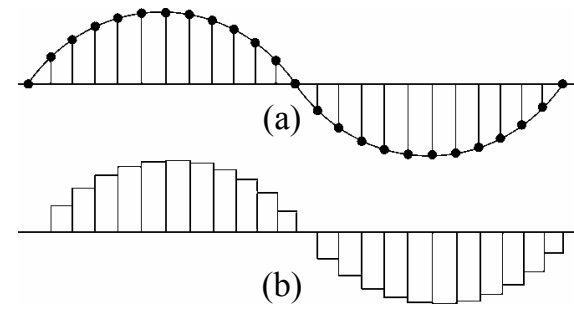

Figura 5. (a) Representação da amostragem do sinal de referência senoidal $\left(m_{s}=24\right)$. (b) Representação da tensão média sintetizada em cada intervalo entre amostras sucessivas.

Com relação à estratégia de modulação e operação do inversor, deve-se garantir que:

4. Os sinais de referência são sintetizados pelo inversor utilizando uma regra previamente definida para as comutações (o que caracteriza a modulação space vector). Observa-se que cada interruptor pode comutar várias vezes em um intervalo de amostragem, desde que isso esteja definido nas regras de comutação;

5. Os interruptores do inversor são considerados ideais, sendo desconsideradas as quedas de tensão, a resistência de condução e os atrasos de comutação;

6. A tensão que alimenta o filtro de saída do inversor é determinada exclusivamente pelo estado de comando dos interruptores e pela tensão no barramento CC (ou derivações do mesmo) do inversor. Essa condição é obtida, em qualquer instante de tempo, se os diodos em anti-paralelo aos interruptores não entrarem em condução.

Com relação ao espectro do sinal modulado resultante, assume-se que:

7. O espectro harmônico das tensões geradas pelo inversor que alimenta o filtro de saída em um ciclo do sinal de referência é formado predominantemente pela componente harmônica fundamental e por componentes harmônicas de alta freqüência. Assume-se que a componente harmônica fundamental está localizada abaixo da freqüência natural do filtro enquanto as componentes harmônicas de alta freqüência estão localizadas acima desta freqüência;

8. É assumida a existência de um distanciamento espectral entre a componente fundamental e a freqüência natural do filtro, suficiente para garantir que variações da resistência de carga não alterarão significativamente 0 valor das componentes harmônicas, tal como discutido na Seção 2. A mesma hipótese é assumida em relação às componentes harmônicas de alta freqüência. Para os casos onde o inversor opera com uma lei de controle, o afastamento espectral da componente fundamental e freqüência natural fica sendo dado pela resposta em freqüência do sistema em malha fechada.;

9. As componentes harmônicas de alta freqüência com amplitude significativa estão todas localizadas em bandas laterais a múltiplos ou sub-múltiplos de $m_{s}$, como ilustrado na Fig.3. Além disso, deve ser válida a aproximação dada em (9) para os primeiros grupos de harmônicas.

Com relação à topologia do filtro de saída empregado, assume-se que:

10. As saídas do filtro são simétricas entre si ou em relação a um ponto comum (ex.: neutro em sistemas trifásicos a quatro fios);

11. Todos os indutores de um dado filtro possuem indutância idêntica e resistências internas desprezíveis. Similarmente, todos os capacitores também possuem capacitâncias idênticas e resistências internas desprezíveis;

12. O filtro de saída deve ser representável na forma de matriz de funções de transferência, considerando como entradas as tensões geradas pelos braços do inversor e como saídas às tensões que alimentam a carga. Essa matriz deverá possuir a forma $|\mathbf{V}[n]|=\left.\mathbf{G}(s)\right|_{s=j \omega_{1} n}|\mathbf{U}[n]|, \quad n=1,2, \ldots$, ou

$$
\left(\begin{array}{c}
\mid V_{1}[n] \\
\mid V_{2}[n] \\
\vdots \\
\mid V_{v}[n]
\end{array}\right)=\left(\begin{array}{cccc}
\left|G_{1}(s)\right| & \left|G_{2}(s)\right| & \cdots & \left|G_{2}(s)\right| \\
\left|G_{2}(s)\right| & \left|G_{1}(s)\right| & \cdots & \left|G_{2}(s)\right| \\
\vdots & \vdots & \ddots & \vdots \\
\left|G_{2}(s)\right| & \left|G_{2}(s)\right| & \cdots & \left|G_{1}(s)\right|
\end{array}\right) \mid\left(\begin{array}{c}
\left|U_{1}[n]\right| \\
\left|U_{2}[n]\right| \\
\vdots \\
\left|U_{v}[n]\right|
\end{array}\right)
$$

onde $V_{1}[n], \ldots V_{v}[n]$ são as tensões de saída cujas THD são especificadas, $\left|U_{1}[n]\right|, \ldots\left|U_{v}[n]\right|$ são as fontes de tensão equivalentes às tensões geradas pelos braços do inversor, $G_{1}(s)$ são as funções de transferência entre as entradas e as saídas diretas e $G_{2}(s)$ são as funções de transferência entre as entradas e as saídas cruzadas, para $s=j \omega_{1} n$.

13. As funções de transferência $G_{1}(s)$ possuem um comportamento passa-baixas, com atenuação de $40 \mathrm{~dB} /$ década nas altas freqüências.

14. As funções de transferência $G_{2}(s)$ ou são nulas ou possuem um comportamento passa-banda, com atenuação de $40 \mathrm{~dB} /$ década nas altas freqüências e com significativa atenuação da componente fundamental em relação à $G_{1}(s)$.

\subsubsection{Etapas para determinação de $n D F_{2}$}

A partir da definição das hipóteses a serem satisfeitas, será apresentada a sistematização, para diversas topologias de inversores, da metodologia descrita na Seção 2. A metodologia utiliza, para o projeto, a seguinte equação:

$$
f_{r}(p)=f_{1} m_{s} \sqrt{\frac{T H D_{v}}{n D F_{2}(p, m)}}
$$


onde $p$ é o índice associado a cada uma das tensões de saída do filtro. A metodologia para a determinação do índice $n D F_{2}(p, m)$ que consiste nas seguintes etapas:

\section{Etapa 1 Determinação do modelo matemático do filtro}

O primeiro passo é a obtenção do modelo nominal do filtro na forma de matriz de funções de transferência, como mostrado em (13). Este modelo é obtido considerando as tensões geradas pelos braços do inversor como fontes ideais de tensão. Já as fontes de saída são consideradas entre os terminais de saída do inversor, como, por exemplo, entre fase-fase e fase-neutro em inversores trifásicos com saída a três fios e a quatro fios, respectivamente.

\section{Etapa 2 Aproximação da resposta em freqüência do filtro por assíntotas}

A partir da matriz de funções de transferência dada em (13), faz-se a aproximação assintótica de $G_{1}(s)$ e $G_{2}(s)$ para as regiões do espectro onde existem componentes harmônicas com magnitudes significativas. Obtém-se, desta forma, as funções de transferência equivalentes $G_{1}{ }^{*}(s)$ e $G_{2}{ }^{*}(s)$, que consistem em uma aproximação do ganho do filtro para a componente fundamental e uma aproximação assintótica, de segunda ordem, para o ganho das freqüências acima da freqüência natural do filtro. Por exemplo, para a $G_{1}(s)$ dada por

$$
G_{1}(s)=\frac{3}{4} \frac{\omega_{r}^{2}\left(s^{2}+2 \zeta \omega_{r} s+\omega_{r}^{2} / 3\right)}{\left(s^{2}+2 \zeta \omega_{r} s+\omega_{r}^{2}\right)\left(s^{2}+2 \zeta \omega_{r} s+\omega_{r}^{2} / 4\right)}
$$

é feita a seguinte aproximação:

$$
\left|G_{1}^{*}(s)\right|=\left\{\begin{array}{c}
1 \\
\frac{3}{4}\left(\frac{\omega_{r}}{s}\right)^{2}, \quad s \gg j \omega_{1}
\end{array}\right.
$$

Após essa etapa, é necessário se descrever $\left|G_{1}{ }^{*}(s)\right|\left|U_{q}[n]\right|$, substituindo-se $s$ por $j \omega_{1} n$ em $\left|G_{1}^{*}(s)\right|$. Por exemplo, (16) fica sendo descrita por

$$
\left|\mathbf{G}_{p, q}{ }^{*}\left(j \omega_{1} n\right)\right|\left|U_{q}[n]\right|=\left\{\begin{array}{c}
\left|U_{q}[1]\right| \\
\frac{3}{4}\left(\frac{f_{r}}{f_{1}}\right)^{2} \frac{1}{n^{2}}\left|U_{q}[n]\right|, n \gg \frac{f_{r}}{f_{1}}, n \in \mathbb{N}^{+}
\end{array}\right.
$$

onde $q$ é o índice associado a cada uma das tensões de entrada do filtro. A aproximação utilizada em $G_{2}(s)$ é semelhante à apresentada para $G_{1}(s)$, com diferença de que para a componente fundamental de $G_{2}{ }^{*}(s)$ o ganho é nulo. É válido mencionar que para essa aproximação ser válida, é necessário que $\left|G_{2}{ }^{*}\left(j \omega_{1}\right)\right|<<\left|G_{1}{ }^{*}\left(j \omega_{1}\right)\right|$.

\section{Etapa 3 Análise da variação da resistência de carga}

Após se determinar a aproximação assintótica, verifica-se a faixa de freqüências onde os erros associados a aproximação são admissíveis. Esta análise é efetuada considerando-se as possíveis variações na resposta em freqüência em função de alterações na resistência de carga. Para tanto, é realizada a comparação entre todos os $G_{1}(s)$ e $G_{2}(s)$ e suas respectivas aproximações $G_{1}{ }^{*}(s)$ e $G_{2}{ }^{*}(s)$, considerando-se variações do coeficiente de amortecimento $0 \leq \zeta \leq 1$, que são os valores mínimo e máximo típicos de projeto. A partir desta análise, determina-se a faixa onde estas variações de $\zeta$ resultam em erros muito grandes ( $>1 \%$ ou $>5 \%$, por exemplo) em relação à aproximação por assíntotas. É importante se salientar que, para a determinação do afastamento entre a freqüência do sinal de referência e a natural do filtro, emprega-se a função de transferência do sistema em malha fechada. Desta forma, assumindo-se que o espectro dos sinais gerados pelo inversor não possua componentes harmônicas dentro dessa faixa de freqüências, se garante que o procedimento de projeto é confiável e independente da resistência de carga.

\section{Etapa 4 Determinação de $\boldsymbol{m}$}

O passo seguinte é a determinação do índice de modulação $m$ do inversor empregado. Este índice é definido entre 0 e 1 , sendo assumido que $m=1$ para o valor máximo possível a ser gerado nas tensões das saídas $V_{x}[s], x=1,2, \ldots, v$, sem sobremodulação. Por exemplo, para o inversor trifásico com saída a quatro fios cujas saídas são dadas como sendo a tensão entre a fase e o neutro, define-se $m$ como sendo $\sqrt{3}\left|U_{x}[1]\right| / E$, pois a máxima tensão de saída de fase possível de ser gerada por esse inversor é $E / \sqrt{3}$. É interessante mencionar que o mesmo vale também para os inversores polifásicos nos quais os sinais modulantes precisam ter magnitudes diferentes entre si para gerar tensões com mesma amplitude na saída.

\section{Etapa 5 Verificação da adequação da estratégia de modulação às hipóteses assumidas}

Dada a estratégia de modulação PWM digital adotada, o próximo passo é verificar se a mesma atende às hipóteses 7 , 8 e 9 . Isso pode ser realizado através da geração matemática do padrão das tensões que foram modeladas como as tensões de entradas do filtro $u_{1}, u_{2}, \ldots, u_{v}$ de forma idênticas a àquelas obtidas no inversor para a estratégia de modulação adotada. Estes padrões devem ser gerados para um ciclo completo dos sinais de referência, tomando-se diversos valores de $m e$ utilizando uma freqüência de amostragem normalizada $m_{s}$ suficientemente alta para 
assegurar erros pequenos nas aproximações assumidas. Após isso, se aplica uma transformada de Fourier nestas formas de onda e se verifica se as hipóteses 7,8 e 9 são atendidas. Se estas hipóteses não forem atendidas, o procedimento apresentado não é válido para esta estratégia de modulação.

\section{Etapa 6 Traçado da curva de $n D F_{2}(p, m)$}

A determinação do índice normalizado $n D F_{2}(p, m)$ é feita através da seguinte equação

$$
n D F_{2}(p, m)=\frac{1}{\mid U_{p}[1]}\left(\sum_{q=1}^{v} \operatorname{sinal}\left(\mathbf{G}_{p, q}(s)\right) W(q)\right)^{\frac{1}{2}}
$$

onde

$$
W(q)=\left\{\begin{array}{l}
\sum_{n=a_{1} m_{s}-b_{1}}^{a_{1} m_{s}+b_{1}}\left[\frac{\left|G_{1}^{*}\left(j \omega_{1} n\right)\right|\left|U_{q}[n]\right|}{a_{1}^{2}}\right]^{2} \\
+\sum_{n=a_{2} m_{s}-b_{2}}^{a_{2} m_{s}+b_{2}}\left[\frac{\left|G_{1}^{*}\left(j \omega_{1} n\right)\right|\left|U_{q}[n]\right|}{a_{2}{ }^{2}}\right]^{2}+\ldots, q=p \\
\sum_{n=a_{1} m_{s}-b_{1}}^{a_{1} m_{s}+b_{1}}\left[\frac{\left.\left|G_{2}^{*}\left(j \omega_{1} n\right)\right|\left|U_{q}[n]\right|\right|^{2}}{a_{1}{ }^{2}}\right]^{2} \\
+\sum_{n=a_{2} m_{s}-b_{2}}^{a_{2} m_{s}+b_{2}}\left[\frac{\left|G_{2}{ }^{*}\left(j \omega_{1} n\right)\right|\left|U_{q}[n]\right|}{a_{2}{ }^{2}}\right]^{2}+\ldots, q \neq p
\end{array}\right.
$$

e $\operatorname{sinal}\left(\mathbf{G}_{p, q}(s)\right)$ é o sinal do ganho de $G_{1}(s)$ e $G_{2}(s)$, respectivamente, na matriz de funções de transferência do filtro. Para $\mathrm{n} D F_{2}(p, m)$ ser real, o resultado do somatório apresentado em (18) deve ser positivo. Os índices $a_{1}, a_{2}, \ldots$ são obtidos a partir dos espectros obtidos para a etapa descrita na etapa 5, sendo que cada um destes índices pode ser distinto para as diferentes entradas $u_{q}$, desde que estes respeitem as hipóteses apresentadas. Considera-se que $b_{1}<0,1 a_{1} m_{s}, b_{2}<0,1 a_{2} m_{s}$, e assim sucessivamente.

A seguir, se obtém os índices normalizado $n D F_{2}(p, m)$ para diversos valores de $m$ empregando-se (18). Posteriormente, traça-se os índices obtidos num gráfico, obtendo-se a curva de $n D F_{2}(p, m)$. É interessante ressaltar que caso as tensões de entrada apresentem um espectro idêntico, o índice $n D F_{2}(p, m)$ será o mesmo para qualquer $p$.

\section{DETERMINAÇÃO DA RELAÇÃO ENTRE AS INDUTÂNCIAS E CAPACITÂNCIAS DO FILTRO}

Nesta seção é apresentado o procedimento para a determinação da relação entre as indutâncias e capacitâncias do filtro e dos parâmetros da lei de controle escolhida, para os casos em que o inversor opera em malha fechada. O procedimento de projeto é válido para as topologias que atendam às hipóteses descritas na seção anterior. A metodologia pode ser utilizada tanto para filtros que alimentam cargas lineares quanto não-lineares. Para o primeiro caso, somente se necessita utilizar as etapas 2, 3, 4 e 5 para se realizar o projeto.

A metodologia apresentada busca a determinação da relação $\mathrm{L} / \mathrm{C}$ que seja a mais atrativa em relação ao custo dos componentes, bem como da lei de controle que consiga atenuar suficientemente as componentes harmônicas que aparecem devido às cargas não-lineares. $\mathrm{O}$ objetivo desta seção é o de se conseguir uma THD de saída menor que o valor estipulado no passo 1 , considerando-se somente o efeito das cargas não-lineares nos harmônicos de saída. As sete etapas de projeto são descritas a seguir.

\section{Etapa 1 Determinação do espectro da corrente de saída da carga não-linear, na pior condição de carga}

Inicialmente, define-se a carga não-linear mais crítica a ser utilizada no inversor a ser projetado. Após a determinação desta carga, se realiza uma simulação desta considerando-se que ela está sendo alimentada por uma fonte de tensão senoidal ideal, ou seja, com impedância nula. A simulação é realizada para um ciclo de referência até a carga entrar em regime permanente caso a mesma seja cíclica, como, por exemplo, os retificadores não-controlados. Obtém-se, então, a forma de onda da corrente na fonte senoidal, dos quais se extrai as componentes harmônicas mais relevantes através de uma transformada rápida de Fourier (FFT). É usual se obter o espectro das harmônicas até a $40^{\mathrm{a}}$ ordem (IEC 62040-3, 1999).

\section{Etapa 2 Determinação inicial dos valores de $L e$ C, a partir de uma função custo}

Para se determinar os valores iniciais de L e C é empregada uma função custo que minimiza o custo da energia reativa, para a componente fundamental, nos indutores $\mathrm{e}$ capacitores do filtro. A função custo para a carga resistiva é dada por:

$$
J(L)=\frac{d}{d t}\left(W \sum_{n} Q_{L}+\sum_{m} Q_{C}\right)
$$


onde $Q_{L}$ e $Q_{C}$ é o somatório das energias reativas nos $n$ indutores e $m$ capacitores, respectivamente, e $W$ é a proporção entre o custo do kVAr indutivo e capacitivo. Para o inversor monofásico, $Q_{L}$ e $Q_{C}$ são dados por:

$$
\begin{gathered}
Q_{L}=\omega_{1} L\left(I_{o_{r m s}}^{2}-\omega_{1} C V_{o_{r m s}}^{2}\right) \\
Q_{C}=\omega_{1} C V_{o_{r m s}}^{2}
\end{gathered}
$$

onde $V_{o r m s}$ é a tensão rms de saída e $I_{o r m s}$ é a corrente rms de saída. Substituindo-se (21) e (22) em (20), chega-se as seguintes equações para $L$ e $C$ :

$$
\begin{gathered}
L=\sqrt{\frac{\frac{V_{o_{r m s}^{2}}^{2}}{\omega_{n}^{2}}\left(W \frac{\omega_{1}^{2}}{\omega_{n}^{2}}+1\right)}{W I_{o_{r m s}}^{2}}} \\
C=\frac{1}{\omega_{n}^{2} L}
\end{gathered}
$$

Para esta análise ser válida, é necessário se garantir que a ondulação de alta freqüência nos componentes reativos seja pequena (menor que $25 \%$ do valor nominal).

\section{Etapa 3 Determinação do valor mínimo de $L$ que atende às especificações de ondulação máxima da corrente nos indutores}

A determinação do valor mínimo para o indutor para uma dada ondulação máxima de corrente no indutor é realizada para se garantir que as perdas no núcleo magnético e nos interruptores não sejam muito elevadas. Além disso, essa limitação é usada para se garantir que o ruído e a emissão de interferência eletromagnética devidos aos elevados $d i / d t$ não sejam excessivos. Usualmente se utiliza como valor máximo uma ondulação de $25 \%$ da corrente de carga nominal pico-a-pico (Botterón et al., 2001). Este valor pode ser obtido através de da seguinte equação:

$$
L=\frac{E}{\delta_{\max } I_{o p}^{2} f_{1} m_{s}} f(m)
$$

onde $\delta_{\max }$ é a máxima ondulação especificada para a corrente no indutor (valores entre 0 e 1) e $I_{o p p}$ é o valor máximo da corrente pico-a-pico de saída, para cargas puramente resistivas.

A função $f(m)$ depende da topologia, da estratégia de modulação e do índice de modulação $m$. Por ser uma relação não-linear, ela é traçada em um gráfico de modo similar ao apresentado das seções anteriores. Para obter esta função, deve-se considerar que a THD de saída é baixa, e que os capacitores são um curto circuito. Obtém-se, então, o modelo deste circuito na forma de equação de estados, no qual as correntes são dadas por:

$$
\mathbf{I}=\int \mathbf{A} \mathbf{U} d t
$$

onde

$$
\mathbf{I}=\left(\begin{array}{c}
i_{L_{1}} \\
\vdots \\
i_{L_{n}}
\end{array}\right), \quad \mathbf{A}=\left(\begin{array}{ccc}
a_{11} & \cdots & a_{1 n} \\
\vdots & \ddots & \vdots \\
a_{n 1} & \cdots & a_{n n}
\end{array}\right), \quad \mathbf{U}=\left(\begin{array}{c}
u_{1}-v_{o_{1}} \\
\vdots \\
u_{m}-v_{o m}
\end{array}\right)
$$

A partir da resolução temporal das integrais se obtém o valor pico-a-pico máximo para as correntes para a estratégia de modulação e o índice de modulação. As curvas de projeto normalizadas são obtidas a partir da seguinte equação:

$$
f(m)=\frac{L f_{1} m_{s}}{E} I_{p p_{\max }}
$$

onde $L, f_{1}, m_{s}$ e $E$ são os valores que foram utilizados no cálculo de $f(m)$ e $I_{p p \_ \text {max }}$ é o valor máximo da corrente picoa-pico obtido.

Caso o valor de $L$ obtido no passo anterior seja inferior ao determinado nesta etapa, o mesmo deve ser aumentado para o valor obtido em (25), mantendo a relação $L$ e $C$ constante. Se o valor de $C$ também não puder ser diminuido, uma solução é a redução da freqüência natural do filtro, voltando-se à etapa 1 da metodologia descrita na Seção 2 .

\section{Etapa 4 Determinação dos parâmetros da lei de controle para esta combinação de parâmetros L e C}

Nesta etapa é realizado o projeto dos parâmetros do controlador, para a lei de controle escolhida pelo projetista, caso o sistema seja controlado em malha fechada. A determinação dos parâmetros do controlador é somente realizada nesta etapa porque eles dependem dos valores de $L$ e $C$ obtidos no passo anterior. Em geral, para a aplicação em questão, as especificações de desempenho estão relacionadas à resposta transitória, tempo de acomodação e sobre-elevação máxima, considerando-se que o inversor opera com carga resistiva nominal.

\section{Etapa 5 Verificação do cumprimento da especificação de sobre-elevação máxima para o degrau de carga}

A verificação da sobre-elevação máxima da tensão de saída é realizada para se garantir as especificações de projeto. Em inversores para UPS, esta especificação é dada pela norma IEC 62040-3 (1999), que especifica a sobre-elevação máxima para diferentes tipos de degraus de carga. Em geral, o caso mais crítico de sobre-elevação ocorre quando 
se faz uma retirada súbida de carga no instante em que ocorre o pico do sinal de referência.

A sobre-elevação na resposta do sistema pode ser obtida através da análise temporal da resposta do inversor ao impulso. Para se realizar esta análise, se considera que a resposta do inversor, incluindo as suas condições iniciais, é dada por:

$$
\mathbf{Y}(z)=\mathbf{G}_{\mathbf{p}}{ }^{*}(z) \mathbf{U}(z)+\mathbf{G}_{\mathbf{p} 0}{ }^{*}(z)
$$

onde $\mathbf{G}_{\mathbf{p}}{ }^{*}(z)$ é a matriz de funções de transferência do inversor e $\mathbf{G}_{\mathbf{p 0}} *(z)$ é a matriz de tranformadas $z$ da resposta transitória do inversor às condições iniciais, respectivamente. Para a obtenção de $\mathbf{G}_{\mathbf{p}} *(z)$ e $\mathbf{G}_{\mathbf{p} 0} *(z)$ se considera a resistência de carga após o degrau de carga, sendo que em $\mathbf{G}_{\mathbf{p 0}}$ *(z) são usados os valores das tensões e correntes nos capacitores $\mathrm{e}$ indutores no instante imediatamente anterior ao degrau de carga. Deve-se ressaltar que em (29) se considera que o degrau de carga está ocorrendo em $t=0$.

Para os casos em que o sistema opera em malha aberta, basta substituir $\mathbf{U}(z)$ por $\mathbf{R}(z)$, sendo $\mathbf{R}(z)$ a matriz de transformadas $\mathrm{z}$ dos sinais senoidais de referência. Já para os casos em que o inversor possui uma lei de controle, deve-se obter as funções de transferência do sistema em malha fechada. É importante se salientar que, para ambos os casos, as senoides descritas em $\mathbf{R}(z)$ devem possuir uma fase inicial correspondente ao instante em que ocorre o degrau de carga.

Para exemplificar, considera-se um inversor monofásico em malha fechada, cuja representação em blocos está mostrada na Fig. 6, submetido a um degrau de carga resistiva de $100 \%$ para $0 \%$ no instante do pico do sinal de referência. Para este caso, se obtém as seguintes $G_{p} *(z)$ e $G_{p 0} *(z)$ :

$$
\begin{gathered}
G_{p}^{*}(z)=\frac{\left(1-\cos \left(\Omega t_{s}\right)\right)(z+1)}{z^{2}-2 z \cos \left(\Omega t_{s}\right)+1} \\
G_{p 0}^{*}(z)=\frac{z^{2} v_{c}\left(t_{1}\right)+z\left(Z i_{L}\left(t_{1}\right) \sin \left(\Omega t_{s}\right)-v_{c}\left(t_{1}\right) \cos \left(\Omega t_{s}\right)\right)}{z^{2}-2 z \cos \left(\Omega t_{s}\right)+1}
\end{gathered}
$$

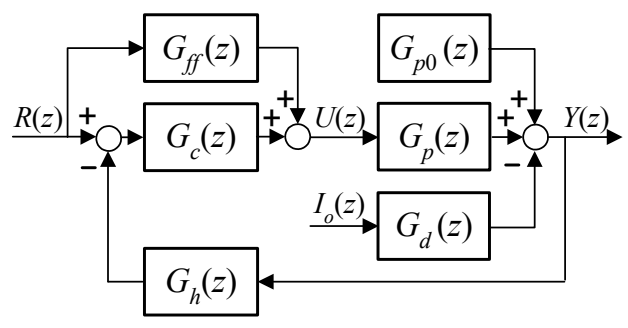

Figura 6. Diagrama em blocos de um inversor PWM monofásico em malha fechada. onde $\Omega=1 / \sqrt{L C}, Z=\sqrt{L / C}$ e $v_{c}\left(t_{1}\right)$ e $i_{L}\left(t_{1}\right)$ são a tensão no capacitor e a corrente no indutor, respectivamente, no instante em que ocorre o degrau de carga.

Tomando-se o restante do sistema de realimentação mostrado na Fig. 6, a resposta deste inversor ao degrau de carga pode ser obtida através da resposta ao impulso a seguinte transformada $\mathrm{z}$ :

$$
Y(z)=\frac{\left(G_{c}(z)+G_{f f}(z)\right) G_{p}{ }^{*}(z) R(z)+G_{p 0}{ }^{*}(z)}{1+G_{h}(z) G_{c}(z) G_{p}{ }^{*}(z)}
$$

onde $R(z)$ é a função de transferência do sinal senoidal de referência. A transformada $\mathrm{z}$ de uma senóide de fase genérica, dada por $r[n]=A \cos \left[\omega_{1} T_{s} n-\phi\right]$, é dada por:

$$
R(z)=\frac{z^{2} A \cos (\phi)-z A \cos \left(\omega_{1} T_{s}+\phi\right)}{z^{2}-2 z \cos \left(\omega_{1} T_{s}\right)+1}
$$

Substituindo-se (30), (31), (33) e mais as funções de transferência do controlador em (32), e posteriormente se aplicando uma resposta ao impulso, se obtém a resposta temporal da saída após o degrau de carga. Comparando-se esta resposta temporal com o sinal de referência se verifica se a sobre-elevação máxima atende às especificações dadas;

Este procedimento pode ser estendido aos conversores trifásicos, lembrando que se deve levar em consideração os efeitos dos acoplamentos do inversor e do controlador. É importante se salientar que não foram consideradas as condições iniciais dos elementos de memória dos filtros e compensadores, sendo estes normalmente de pequena influência em momentos subseqüentes a grandes degraus de carga. Na prática, é muito mais simples e rápido se verificar a sobre-elevação máxima através da simulação do inversor, principalmente para inversores trifásicos e acoplados, nos quais a complexidade matemática do modelo aumenta significativamente.

\section{Etapa 6 Obtenção da impedância de saída do inversor para as freqüências das componentes harmônicas típicas}

O cálculo da impedância de saída para o conversor é realizado obtendo-se o modelo do inversor considerando-se a corrente de carga como uma entrada do sistema. Assim, obtém-se um sistema de funções de transferência na seguinte forma:

$$
\mathbf{Y}(z)=\mathbf{G}_{\mathbf{p}}(z) \mathbf{U}(z)+\mathbf{G}_{\mathbf{d}}(z) \mathbf{I}_{\mathbf{o}}(z)
$$

onde $\mathbf{G}_{\mathbf{p}}(z)$ e $\mathbf{G}_{\mathbf{d}}(z)$ são a matrizes de funções de transferência entre as entradas e saídas e entre as correntes de carga $\mathbf{I}_{\mathbf{0}}(z)$ e as saídas do inversor, respectivamente. Considera-se, para ambas matrizes de transferência, que o 
inversor está operando sem carga. Assim, a impedância da saída $p$ em relação a uma corrente de carga associada a uma entrada $q$ é dada por:

$$
Z_{p, q}(\omega)=\left|\frac{Y_{p}(z)}{I_{o q}(z)}\right|_{z=e^{j \omega T_{S}}}=\left|G_{d p, q}(z)\right|_{z=e^{j \omega T_{S}}}
$$

Após serem obtidas as equações para as impedâncias de saídas, calcula-se as impedâncias para as principais componentes harmônicas de baixa freqüência da corrente de saída.

Para exemplificar, toma-se o inversor monofásico com a lei de controle representada na Fig. 6. A impedância de saída deste inversor em malha fechada é dada por:

$$
Z(\omega)=\left|\frac{G_{d}\left(e^{j \omega T_{s}}\right)}{1+G_{h}\left(e^{j \omega T_{s}}\right) G_{c}\left(e^{j \omega T_{s}}\right) G_{p}\left(e^{j \omega T_{s}}\right)}\right|
$$

A partir de (36) se calcula, então, a impedância de saída para $\omega=2 \pi f_{1}, 2\left(2 \pi f_{1}\right), 3\left(2 \pi f_{1}\right), \ldots$.

\section{Etapa 7 Obtenção da estimativa da THD de saída em função da amplitude das componentes harmônicas da corrente e da impedância de saída}

Nesta etapa é calculada uma estimativa da THD de saída do inversor com a carga não-linear. Este cálculo é realizado empregando a eq. (1), na qual as harmônicas de tensão na saída são estimadas a partir do produto entre as harmônicas de corrente de saída, obtidas na etapa 1, e a impedância de saída nestas freqüências, calculadas na etapa 6. Assim, a THD para uma saída $p$ é obtida através da seguinte equação:

$$
T H D_{p}=\frac{1}{V_{p}\left(\omega_{1}\right)} \sum_{q} \sqrt{\sum_{n=1}^{\infty}\left(Z_{p, q}\left(n \omega_{1}\right) I_{o p, q}\left(n \omega_{1}\right)\right)^{2}}
$$

onde $V_{p}\left(\omega_{1}\right)$ é a amplitude da componente fundamental da tensão de saída.

Deve-se ressaltar que (37) oferece apenas uma estimativa da THD da tensão de saída, pois uma margem de erro superior a $50 \%$ pode existir entre esta e o valor real da THD. Esse erro existe porque o espectro das correntes nas cargas não-lineares depende da forma de onda da tensão de saída, o que é desconsiderado em (37). Por exemplo, para cargas do tipo retificadores não-controlados com filtro capacitivo, Manssor et al., (1995) mostram que a THD da corrente tende a diminuir com afundamentos de tensão. Desta forma, a THD estimada tem tendência a ser sempre maior que o valor real, pois quanto maior o afundamento devido à carga não-linear, menor a distorção na mesma.
Logo, pode-se concluir que a THD estimada será mais próxima do valor correto quanto menor for a THD da tensão de saída.

Devido a estes motivos, estipulou-se utilizar uma margem de erro de $75 \%$. Assim, se o valor estimado da THD devido às harmônicas de baixa freqüência for maior que $175 \%$ do valor máximo especificado para a THD (THD $>8,75 \%$ para $\mathrm{THD}_{\text {máx }}=5 \%$ ), ele provavelmente não atenderá à norma. Neste caso, deve-se diminuir o valor de L e voltar à etapa 3 . Se não for possível por violar a condição dada na etapa 3 , a única solução é manter L constante e aumentar-se o valor de $\mathrm{C}$ e retornar-se novamente a etapa 2, descrita na Seção 2.

\section{Etapa 8 Verificar o valor da THD de saída através de simulação}

Como não há uma maneira de se determinar com precisão a THD de saída com vários tipos de carga não-linear, uma maneira de se obter uma estimativa mais precisa é através de simulações computacionais. Provavelmente, nesta etapa, o valor da THD já esteja bastante próximo do valor especificado. Caso esteja dentro dos limites especificados, ou com valor muito inferior, deve-se diminuir o valor de $\mathrm{L}$ e voltar à etapa 3 . Se não for possível por violar a condição dada na etapa 3 , a única solução é manter L constante e aumentar-se o valor de $\mathrm{C}$ e retornar-se novamente a etapa 2 , descrita na Seção 2.

\section{EXEMPLOS DE PROJETO DE FILTROS E RESULTADOS EXPERIMENTAIS}

Nesta seção são apresentados três exemplos de projeto, para inversores distintos, empregando a metodologia generalizada proposta. O primeiro exemplo mostrará o projeto do filtro para um inversor monofásico alimentando carga não-linear. Os outros dois exemplos apresentaram a derivação da metodologia para obtenção do índice $n D F_{2}(p, m)$ para inversores trifásicos a três fios e quatro fios. Alguns resultados experimentais serão incluídos para demonstração da metodologia proposta.

\subsection{Projeto do filtro LC para um inversor monofásico com carga não-linear}

Este exemplo apresenta a descrição do projeto de um filtro LC monofásico para o inversor ponte completa mostrado na Fig. 1 com modulação PWM centrada de três níveis. $\mathrm{O}$ inversor possui as seguintes especificações: $V_{o}=110 \mathrm{~V}_{\mathrm{rms}}$, $f_{1}=60 \mathrm{~Hz}, m_{s}=167, E=200 \mathrm{~V}, S=1 \mathrm{kVA}$, sendo para o filtro especificado que $W=1$ e $\delta_{\max }=0,4$. A carga a ser utilizada neste inversor é do tipo não-linear, sendo empregada a carga teste especificada pela norma IEC 62040-3 (1999). É também adotada esta norma para a especificação de sobre- 
elevação máxima para degraus de carga, sendo que este inversor deve atender aos requisitos da classe 2. A carga não-linear empregada nos testes, descrita por esta norma, é um retificador não-controlado com filtro capacitivo, cujos parâmetros, para o inversor utilizado, são os seguintes: $R_{s}=0,48 \Omega, \quad R_{1}=28 \Omega$ e $C=4700 \mu \mathrm{F}$. O inversor deverá apresentar uma $T H D<8 \%$ para esta carga não-linear. Vale ressaltar que este projeto não visa atender a todos os requisitos dados por esta norma, tais como a limitação especifíca da amplitude de determinadas harmônicas.

Inicia-se o projeto com determinação da porcentagem de THD de saída que será devido às harmônicas de alta e baixa freqüência. Neste projeto, estipulou-se que dos $8 \%$ da THD de saída, $1 \%$ corresponde às harmônicas de alta freqüência e o restante, $7 \%$, às harmônicas de baixa freqüência. A partir destes valores se determina a freqüência natural do filtro e a relação dos parâmetros L e C, como segue.

\subsubsection{Determinação da freqüência natural do filtro}

\subsubsection{Obtenção do índice $n D F_{2}(m)$}

As etapas para obtenção de $n D F_{2}(m)$, para a modulação space vector apresentada na Fig. 2, são as seguintes:

Etapa 1: Determinação do modelo do filtro do inversor:

$$
\begin{aligned}
& V_{a b}[n]=\left|G_{1}(s)\right|_{s=j \omega_{1} n} U_{a b}[n] \Rightarrow V_{1}[n]=\left|G_{1}(s)\right|_{s=j \omega_{1} n} U_{1}[n] \\
& G_{1}(s)=\frac{\omega_{n}{ }^{2}}{s^{2}+2 \zeta \omega_{n}+\omega_{n}{ }^{2}}, \quad \omega_{n}=\frac{1}{\sqrt{L C}}, \quad \zeta=\frac{1}{2 R} \sqrt{\frac{L}{C}}
\end{aligned}
$$

Etapa 2: Aproximação assintótica:

$$
\left|\mathbf{G}_{1,1}{ }^{*}\left(j \omega_{1} n\right)\right|\left|U_{1}[n]\right|=\left\{\begin{array}{c}
\mid U_{1}[1] \quad, n=1 \\
\left(\frac{f_{r}}{f_{1}}\right)^{2} \frac{1}{n^{2}}\left|U_{1}[n]\right|, n \gg \frac{f_{r}}{f_{1}}, n \in \mathbb{N}^{+}
\end{array}\right.
$$

Etapa 3: A análise da aproximação por assíntotas diante de variações na resistência de carga, para este filtro, é a mostrada anteriormente na Fig. 4. Verifica-se que os erros que ocorrem devido à variações de carga são suficientemente pequenos para $\omega>5 \omega_{r}$ e $\omega<0,1 \omega_{r}$ (quando operando em malha aberta). Logo, não devem existir componentes harmônicas no sinal de $u_{a b}$ dentro desta faixa de freqüências, para que a aproximação seja válida.

Etapa 4: Determinação de $m$. Para este inversor, a máxima amplitude da senóide de saída possível de ser gerada é igual a $E, \log m=U_{a b}[1] / E$.

Etapa 5: Verificação da adequação da estratégia de modulação com as hipóteses assumidas. Realizando-se a
TABELA I. AMPLITUDE DAS PRINCIPAIS HARMÔNICAS DA CORRENTE DE SAÍDA COM A CARGA NÃO-LINEAR UTILIZADA

\begin{tabular}{cccccc}
\hline$n$ & $I_{o}(A)$ & $n$ & $I_{o}(A)$ & $n$ & $I_{o}(A)$ \\
\hline 1 & 9,91 & 15 & 0,70 & 29 & 0,20 \\
\hline 3 & 8,52 & 17 & 0,24 & 31 & 0,17 \\
\hline 5 & 6,15 & 19 & 0,20 & 33 & 0,03 \\
\hline 7 & 3,49 & 21 & 0,36 & 35 & 0,11 \\
\hline 9 & 1,21 & 23 & 0,26 & 37 & 0,12 \\
\hline 11 & 0,36 & 25 & 0,06 & 39 & 0,05 \\
\hline 13 & 0,86 & 27 & 0,15 & 41 & 0,04 \\
\hline
\end{tabular}

transformada rápida de Fourier de $u_{a b}$ obtido a partir de uma senóide de freqüência $f_{1}$ modulada com uma freqüência $f_{s}$, chega-se a um espectro com a forma apresentada na Fig.3(b). Neste espectro se verifica que esta resposta espectral atende as hipóteses 7, 8 e 9 da Seção 3.2.1.

Etapa 6: Traçado da curva de $n D F_{2}(p, m)$. Esta curva é obtida a partir de (18), que neste caso fica igual a (11), sendo $a_{1}=2 m_{s}, a_{2}=4 m_{s}, a_{3}=6 m_{s}$ e $b_{1}=5, b_{2}=10, b_{3}=15$. Os grupos de harmônicas de freqüências maiores que estas podem ser desconsiderados, uma vez que estas harmônicas são sensivelmente atenuadas pelo filtro. Com esta informação do espectro de freqüências e da estratégia de modulação utilizada, chega-se a curva para o fator $n D F_{2}(p, m)$, mostrada na curva "Monofásico" da Fig. 14.

\subsubsection{Determinação da freqüência natural}

A freqüência natural do filtro é calculada usando-se a expressão dada em (14). Para se obter $f_{r}$, inicialmente se obtém o valor de $m$ :

$$
m=\frac{V o}{E}=\frac{110 \sqrt{2}}{200}=0,778 .
$$

Dado o valor de $m$, obtém-se $n D F_{2}(m)$ na curva dada na Fig. 14 para a estratégia de modulação monofásica. Para este caso, $n D F_{2}(0,778)=0,69$ e $T H D_{v}=1 \%$, que aplicados em (14) resulta em $f_{r}=1206,26 \mathrm{~Hz}$.

Para verificar a metodologia, realizou-se uma simulação usando-se um inversor ideal em malha aberta, operando sem carga, onde $L=0,8 \mathrm{mH}$ e $C=20 \mu \mathrm{F}$. Obteve-se que $T H D_{v}=0,971 \%$, que é um valor muito próximo do esperado. Experimentalmente foi empregado um osciloscópio com capacidade de realizar 2000 aquisições em um ciclo do sinal de referência. Os dados obtidos no osciloscópio foram transferidos para um microcomputador, onde foi calculada a $T H D_{v}=0,78 \%$. Foram descosideradas, neste cálculo, as harmônicas de baixa freqüência (até a $40^{\mathrm{a}}$ ordem) que se originam das não-idealidades do inversor, resultando em uma $T H D_{v}=2,59 \%$. As Fig.7(a)-(c) mostram, respectivamente, o padrão PWM amostrado utilizado, a tensão de saída e o espectro da tensão de saída, para este exemplo. 


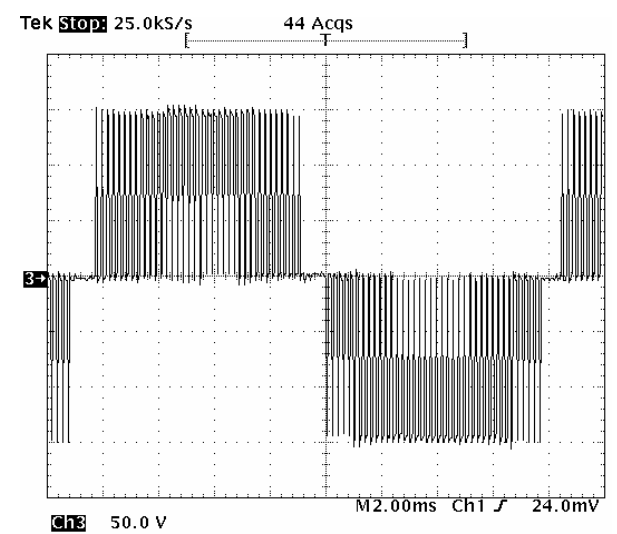

(a)

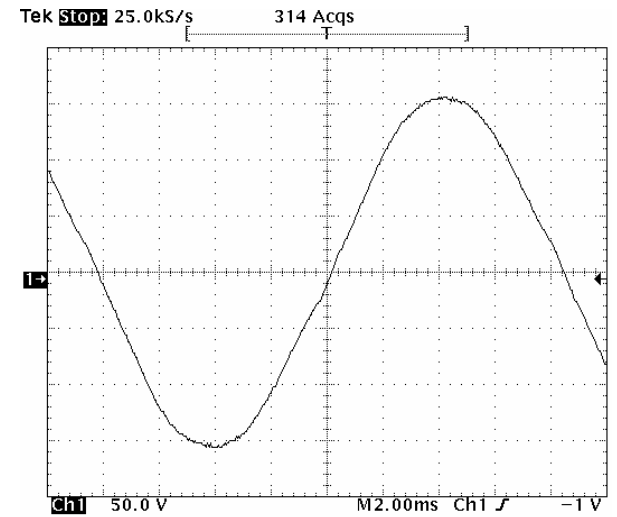

(b)

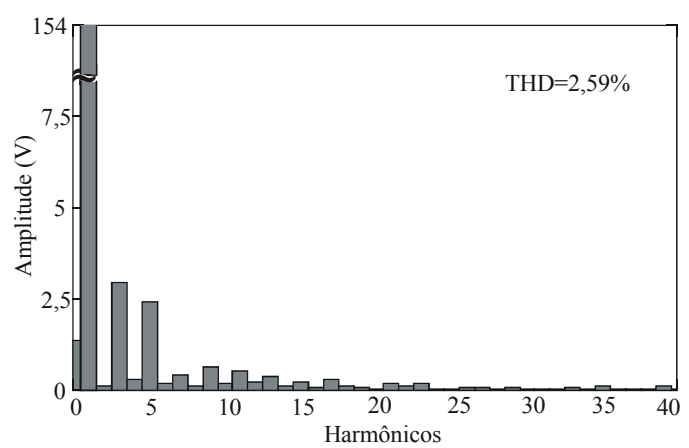

(c)

Figura 7. Formas de ondas experimentais obtidas para o inversor monofásico ponte completa, com o filtro LC projetado na Seção 4.1, para uma modulação PWM centrada

(a) Tensão $u_{a b}$ gerada pelo inversor $(50 \mathrm{~V} / \mathrm{div}, 2 \mathrm{~ms} / \mathrm{div})$

(b) Tensão $v_{a b}$ na saída do filtro $(50 \mathrm{~V} / \mathrm{div}, 2 \mathrm{~ms} / \mathrm{div})$

(c) Espetro da tensão $v_{a b}$ na saída do filtro $(50 \mathrm{~V} / \mathrm{div}, 2 \mathrm{~ms} / \mathrm{div})$

\subsubsection{Determinação dos parâmetros L e C}

A determinação dos parâmetros $\mathrm{L}$ e C é obtida através das etapas dada na Seção 4, descritas a seguir.

Etapa 1: O espectro da corrente da carga de teste foi obtido em um software de simulação de circuitos eletrônicos. As harmônicas de corrente para as principais harmônicas estão mostradas na Tabela 1

Etapa 2: Os valores iniciais para L e C são obtidos através de (23) e (24), onde $W=1$. Obtém-se os seguintes valores: $L=1,60 \mathrm{mH}$ e $C=10,89 \mu \mathrm{F}$. Para se obter um valor comercial de capacitância, utilizou-se $C=10 \mu \mathrm{F}$ e $L=1,75 \mathrm{mH}$.

Etapa 3: O valor mínimo da indutância é dado por (25), na qual $\delta_{\max }=0,4$ e $f(0,778)=0,247$ para a seqüência de comutação em três níveis utilizada. Obteve-se que $L \geq 480 \mu \mathrm{H}$, o que resultaria em $C=36,27 \mu \mathrm{F}$, se a relação $\mathrm{LC}$ for mantida constante. Observa-se que os valores obtidos na etapa 2 atendem a esta restrição, podendo, por isso, serem utilizados. Simulando-se, obteve-se uma ondulação de corrente no indutor de $\delta=40,73 \%$ para a corrrente de carga nominal de 13A.

Etapa 4: Nesta etapa escolheu-se os parâmetros da lei de controle. A lei de controle escolhida foi a proporcionalderivativa (PD) com ação de alimentação à frente (feedforward). Esta lei de controle é dada por:

$$
u(k+1)=r(k+1)+k_{1} e(k)+k_{2} e(k-1) .
$$

onde $k$ é o índice da amostra e $e$ é o sinal de erro entre a referência $r(k)$ e a saída $y(k)$. Considerando a operação em carga resistiva nominal e que os parâmetros de projeto são $\zeta=0,4, \omega_{p}=1,1 \omega_{n}$, chegou-se a parâmetros $k_{1}$ e $k_{2}$ que tornam a lei de controle instável. Por esse motivo, optou-se pelo retorno à etapa 2 e pela busca de uma nova combinação dos parâmetros $L$ e $C$.

Etapas 2 e 3: Escolheu-se novos parâmetros, que são sempre obtidos aumentando-se o valor da capacitância e reduzindo a indutância. Neste caso, empregou-se $C=20 \mu \mathrm{F}$ e $L=0,8 \mathrm{mH}$, que atendem à restrição imposta na etapa 3 .

Etapa 4: Os parâmetros da nova lei de controle são $k_{1}=$ 0,085 e $k_{2}=-0,103$.

Etapa 5: O sistema em malha fechada atende às especificações impostas para degrau de carga resistiva nominal de $100 \%$ para $20 \%$, conforme exigido pela especificação do projeto.

Etapa 6: A impedância de saída foi calculada para as principais harmônicas, cujos resultados obtidos são mostrados na Tabela II. Observa-se que a impedância para algumas harmônicas é muito elevada, o que gera distorções significativas na tensão de saída.

Etapa 7: Calculou-se, então, a THD com carga não-linear, obtendo-se $T H D_{v}=16,96 \%$. Como descrito anteriormente, este filtro não deve estar atendendo a especificação, pois $16,96 \%>12,25 \%(1,75 \cdot 7 \%)$. 
TABELA II. IMPEDÂNCIA DE SAÍDA DO INVERSOR PARA CADA HARMÔNICA, PARA C $=20 \mu \mathrm{F}, \mathrm{L}=0,8 \mathrm{MH}, \mathrm{K}_{1}=-0,085 \mathrm{E} \quad \mathrm{K}_{2}=-0,103$.

\begin{tabular}{cccccc}
\hline$n$ & $Z[\Omega]$ & $n$ & $Z[\Omega]$ & $n$ & $Z[\Omega]$ \\
\hline 1 & 0,27 & 15 & 6,94 & 29 & 5,47 \\
\hline 3 & 0,81 & 17 & 9,42 & 31 & 4,68 \\
\hline 5 & 1,39 & 19 & 11,94 & 33 & 4,08 \\
\hline 7 & 2,05 & 21 & 12,20 & 35 & 3,63 \\
\hline 9 & 2,82 & 23 & 10,21 & 37 & 3,27 \\
\hline 11 & 3,81 & 25 & 8,12 & 39 & 2,98 \\
\hline 13 & 5,11 & 27 & 6,56 & 41 & 2,86 \\
\hline
\end{tabular}

Etapa 8: Com o propósito de ratificar as previsões, foi realizada uma simulação do inversor. A simulação foi efetuada sem utilizar-se modulação PWM, e resultou em $T H D_{v}=8,93 \%$. Por esse motivo, optou-se por se retornar à etapa 2 e se optar por uma outra combinação dos parâmetros $L$ e $C$.

Etapas 2 e 3: Escolheu-se novos parâmetros para o filtro LC: $C=35 \mu \mathrm{F}$ e $L=0,5 \mathrm{mH}$. Os parâmetros atendem à restrição imposta na etapa 3 .

Etapa 4: Os parâmetros da nova lei de controle são $k_{1}=-0,204$ e $k_{2}=-0,121$.

Etapa 5: O sistema em malha fechada atende às especificações impostas para degrau de carga resistiva nominal.

Etapa 6: A impedância de saída foi calculada para as principais harmônicas, cujos resultados obtidos estão mostrados na Tabela III.

Etapa 7: Calculou-se, então, a THD com carga não-linear, obtendo-se $T H D_{v}=10,60 \%$. Neste caso, o filtro deve atender a especificação, pois $10,60 \%<12,25 \%$.

Etapa 8: Com o propósito de ratificar as previsões, foi realizada uma simulação, efetuada sem a modulação PWM, que resultou em $T H D_{v}=6,56 \%$. Incluindo-se a modulação PWM, se obtém uma $T H D_{v}=6,33 \%$, que é menor que o valor previsto $\left(T H D_{v}=7,56 \%\right)$ devido aos efeitos não-

TABELA III. IMPEDÂNCIA DE SAÍDA DO INVERSOR PARA CADA HARMÔNICA, PARA $\mathrm{C}=35 \mu \mathrm{F}, \mathrm{L}=0,5 \mathrm{MH}, \mathrm{K}_{1}=-0,204 \mathrm{E} \quad \mathrm{K}_{2}=-0,121$.

\begin{tabular}{cccccc}
\hline$n$ & $Z[\Omega]$ & $n$ & $Z[\Omega]$ & $n$ & $Z[\Omega]$ \\
\hline 1 & 0,25 & 15 & 0,95 & 29 & 0,37 \\
\hline 3 & 0,84 & 17 & 0,79 & 31 & 0,34 \\
\hline 5 & 1,64 & 19 & 0,67 & 33 & 0,32 \\
\hline 7 & 2,28 & 21 & 0,58 & 35 & 0,29 \\
\hline 9 & 2,02 & 23 & 0,51 & 37 & 0,27 \\
\hline 11 & 1,52 & 25 & 0,45 & 39 & 0,26 \\
\hline 13 & 1,18 & 27 & 0,41 & 41 & 0,25 \\
\hline
\end{tabular}

lineares da ondulação de alta-freqüência sobre a carga, desprezados na análise efetuada

\subsubsection{Resultados experimentais}

Para verificação experimental do procedimento de projeto proposto foi implementado o inversor da Fig. 1 utilizandose o filtro projetado acima. A $T H D_{v}$ obtida experimentalmente, com o inversor operando sem carga, foi de $7,12 \%$, valor este muito próximo ao valor $6,56 \%$ obtido no procedimento de projeto. A Fig. 8 mostra, experimentalmente, a tensão de saída e a corrente de carga para esta topologia, além do espectro da tensão de saída.

Para ilustrar a metodologia para a determinação da freqüência natural foram realizados vários testes, em malha aberta, para diferentes combinações de parâmetros $L, C$ e $f_{s}$. As $T H D_{v}$ obtidas experimentalmente nestes testes foram comparadas com os valores previstos, pela metodologia proposta, através da eq.(12). Os resultados comparativos são apresentados na Tabela IV, e demonstram a validade da metodologia.

\subsection{Determinação da freqüência natural do filtro para inversores trifásicos a três fios}

Este exemplo apresenta a descrição do procedimento para obtenção da freqüência natural de um de um filtro LC trifásico a três fios. A representação da topologia do inversor está ilustrada na Fig. 9, sendo que os detalhes da modulação space vector utilizada estão mostrados no Apêndice A.1.

\subsubsection{Obtenção do índice $n D F_{2}(p, m)$}

As etapas para obtenção de $n D F_{2}(m)$, para esta modulação space vector, são as seguintes:

Etapa 1: Determinação do modelo do filtro do inversor. Considerando-se que o filtro de saída tem os capacitores ligados em Y, o modelo do filtro de saída é dado por:

TABELA IV. COMPARAÇÕES ENTRE A THD CALCULADA E OBTIDA EXPERIMENTALMENTE PARA O INVERSOR MONOFÁSICO COM MODULAÇÃO PWM CENTRADO

\begin{tabular}{|c|c|c|c|c|c|c|}
\hline $\begin{array}{l}\text { Número } \\
\text { do teste }\end{array}$ & $m$ & $f_{\mathrm{s}}(\mathrm{kHz})$ & $L(\mu \mathrm{H})$ & $C(\mu \mathrm{F})$ & $\begin{array}{l}T H D_{v}(\%) \\
\text { calculada }\end{array}$ & $\begin{array}{c}T H D_{v}(\%) \\
\text { medida }\end{array}$ \\
\hline 1 & 1 & 4,98 & 250 & 60 & 2,86 & 2,93 \\
\hline 2 & 1 & 4,98 & 500 & 60 & 1,43 & 1,41 \\
\hline 3 & 1 & 2,52 & 250 & 60 & 11,17 & 14,85 \\
\hline 4 & 1 & 2,52 & 500 & 60 & 5,58 & 6,23 \\
\hline 5 & 0,5 & 4,98 & 250 & 60 & 7,35 & 7,54 \\
\hline 6 & 0,5 & 4,98 & 500 & 60 & 3,68 & 3,63 \\
\hline 7 & 0,5 & 2,52 & 250 & 60 & 28,72 & 37,8 \\
\hline 8 & 0,5 & 2,52 & 500 & 60 & 14,36 & 15,85 \\
\hline
\end{tabular}




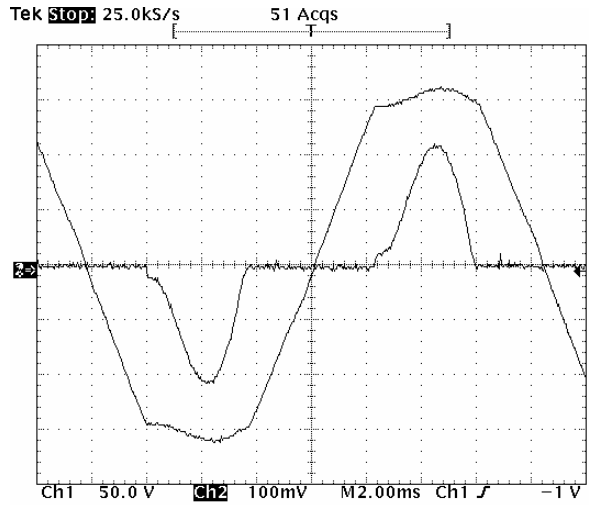

(a)

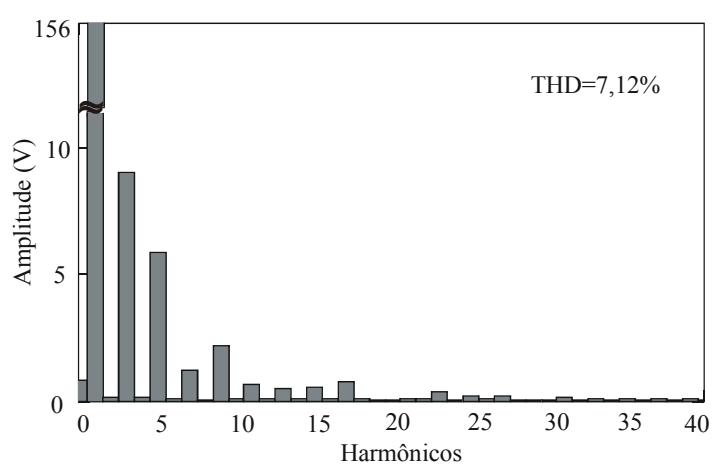

(b)

Figura 8. Formas de ondas experimentais obtidas para o inversor monofásico, com carga não-linear, com o filtro LC projetado.

(a) Tensão $v_{a b}$ e corrente $i_{o}(50 \mathrm{~V} / \mathrm{div}, 10 \mathrm{~A} / \mathrm{div}, 2 \mathrm{~ms} / \mathrm{div})$

(b) Espectro da tensão $v_{a b}(50 \mathrm{~V} / \mathrm{div}, 2 \mathrm{~ms} / \mathrm{div})$

$$
\begin{aligned}
& \left(\begin{array}{l}
\mid V_{a b}[n] \\
\left|V_{b c}[n]\right| \\
\left|V_{c a}[n]\right|
\end{array}\right)=\left.\left(\begin{array}{ccc}
\left|G_{1}(s)\right| & 0 & 0 \\
0 & \left|G_{1}(s)\right| & 0 \\
0 & 0 & \left|G_{1}(s)\right|
\end{array}\right)\right|_{s=j \omega_{1} n}\left(\begin{array}{l}
\left|U_{a b}[n]\right| \\
\left|U_{b c}[n]\right| \\
\left|U_{c a}[n]\right|
\end{array}\right) \\
& G_{1}(s)=\frac{\omega_{r}^{2}}{s^{2}+2 \zeta \omega_{r}+\omega_{r}{ }^{2}}, \quad \omega_{r}=\frac{1}{\sqrt{L C}}, \quad \zeta=\frac{1}{2 R} \sqrt{\frac{L}{C}},
\end{aligned}
$$

$\left[V_{1}[n] V_{2}[n] V_{3}[n]\right]^{\mathrm{T}}=\left[V_{a b}[n] V_{b c}[n] V_{c a}[n]\right]^{\mathrm{T}} \mathrm{e}$

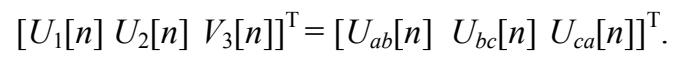

É importante ressaltar que as entradas $u_{a b}, u_{b c}$ e $u_{c a}$ são

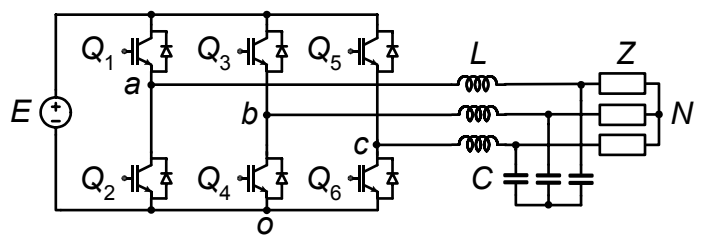

Figura 9. Inversor de tensão trifásico de três braços a três fios com filtro LC de segunda ordem. linearmente dependentes, pois $u_{a b}+u_{b c}+u_{c a}=0$ (o mesmo vale para as saídas). Logo, é necessário se representar apenas duas das equações, pois a terceira será uma combinação linear das outras duas.

Caso os capacitores estivessem ligados em $\Delta$, o modelo dinâmico seria similar ao apresentado por (23) e (24), sendo neste caso:

$$
\omega_{r}=\frac{1}{\sqrt{3 L C}}, \quad \zeta=\frac{1}{2 \sqrt{3} R} \sqrt{\frac{L}{C}}
$$

Etapa 2: Aproximação assintótica:

$$
\left|\mathbf{G}_{1,1}{ }^{*}\left(j \omega_{1} n\right)\right|\left|U_{1}[n]\right|=\left\{\begin{array}{c}
\left|U_{1}[1]\right| \quad, n=1 \\
\left(\frac{f_{r}}{f_{1}}\right)^{2} \frac{1}{n^{2}}\left|U_{1}[n]\right|, n \gg \frac{f_{r}}{f_{1}}, n \in \mathbb{N}^{+}
\end{array}\right.
$$

Etapa 3: A análise da aproximação assintótica diante de variações na resistência de carga é idêntica a apresentada na Seção 4.1 .

Etapa 4: Determinação de $m$. Para este inversor, a máxima amplitude da senóide de saída possível de ser gerada sem sobremodulação é igual a $E, \operatorname{logo} m=U_{a b}[1] / E=U_{1}[1] / E$.

Etapa 5: Verificação da adequação da estratégia de modulação com as hipóteses assumidas. Realizando-se a transformada rápida de Fourier de $u_{a b}$ e $u_{b c}$ obtida a partir de uma senóide de freqüência $f_{1}$ modulada com uma freqüência $f_{s}$, chega-se a um espectro com a forma similar à apresentada na Fig.3, onde se verifica que esta resposta espectral atende às hipóteses 7, 8 e 9 assumidas.

Etapa 6: Traçado da curva de $n D F_{2}(p, m)$. Esta curva é obtida a partir de (18), sendo $a_{1}=m_{s}, a_{2}=2 m_{s}, a_{3}=3 m_{s}$, e $b_{1}=5$, $b_{2}=10, b_{3}=15$. Os grupos de harmônicas de freqüências maior que estas podem ser desconsiderados, uma vez que estas harmônicas são sensivelmente atenuadas pelo filtro. Com esta informação do espetro de freqüências da estratégia de modulação utilizada para o inversor trifásico a três fios se traça a curva de projeto, mostrada na Fig. 14. Como os sinais gerados pelo inversor são simétricos, $n D F_{2}(p, m)=n D F_{2}(m)$.

\subsubsection{Resultados experimentais}

Neste exemplo, estipulou-se as seguintes especificações para o projeto do filtro: $T H D_{v}=3 \%, f_{1}=60 \mathrm{~Hz}, f_{s}=4,98 \mathrm{kHz}$, $m=1$.

A determinação da freqüência natural do filtro então é efetuada usando (14), na qual $m_{s}=f_{s} / f_{1}=83$ e o fator de distorção $n D F_{2}=0,42$ é obtido na Fig.14 para $m=1$. Aplicando-se estes dados em (14), obteve-se a freqüência natural não-amortecida de $f_{r}=1331 \mathrm{~Hz}$. Para verificação experimental do procedimento de projeto foi implementado o inversor com o filtro projetado. Escolheu-se para a implementação um indutor de $L=250 \mu \mathrm{H}$, sendo o capacitor 


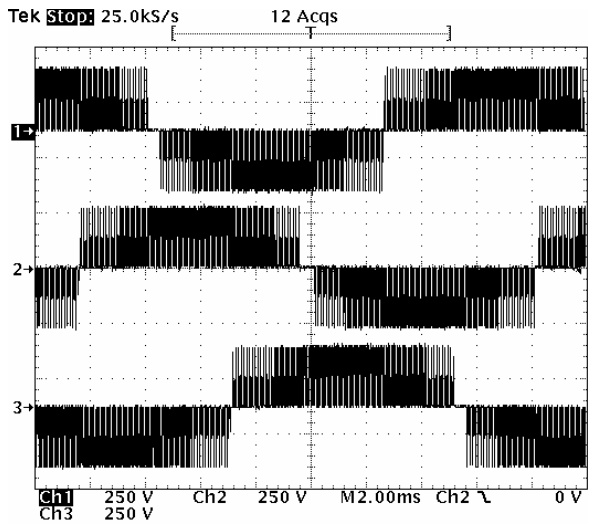

(a)

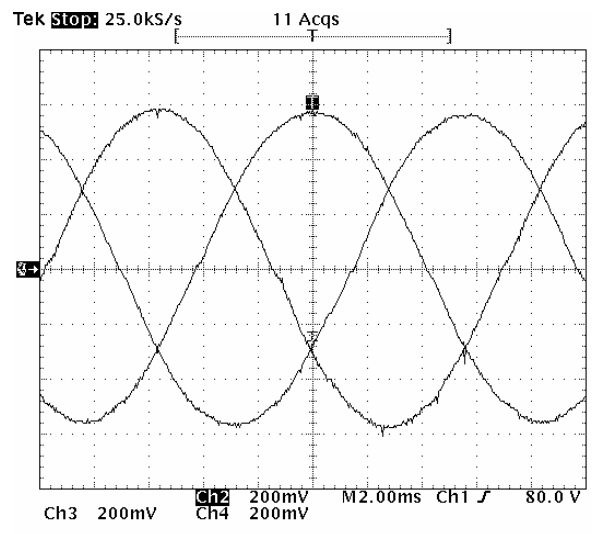

(b)

Figura 10. Formas de onda experimentais obtidas para o inversor trifásico a três fios com estratégia de modulação space vector com o filtro de segunda-ordem mostrado na Seção 5.2.2.

(a) Tensão $u_{a b}$ gerada pelo inversor $(250 \mathrm{~V} / \mathrm{div}, 2 \mathrm{~ms} / \mathrm{div})$.

(b) Tensão $v_{a b}$ na saída do filtro $(50 \mathrm{~V} / \mathrm{div}, 2 \mathrm{~ms} / \mathrm{div})$.

determinado pela relação $\omega_{r}{ }^{2}=1 / L C$, o que resultou em $C=57,19 \mu \mathrm{F}$, sendo utilizado $C=60 \mu \mathrm{F}$, que é a capacitância mais próxima comercialmente existente. A $T H D_{v}$ obtida experimentalmente, com o inversor operando sem carga, foi de 2,77\%. As Fig.10(a) e Fig.10(b) mostram, respectivamente, o padrão space vector utilizado e a tensão entre as fases de saída para esta topologia.

De maneira similar à descrita na seção 5.1.3, determinou-se

TABELA V. COMPARAÇÕES ENTRE A THD CALCULADA E OBTIDA EXPERIMENTALMENTE PARA O INVERSOR TRIFÁSICO COM TRÊS BRAÇOS A

\begin{tabular}{ccccccc}
\hline $\begin{array}{c}\text { Número } \\
\text { do teste }\end{array}$ & $m$ & \multicolumn{6}{c}{$f_{\mathrm{s}}(\mathrm{kHz})$} & $L(\mu \mathrm{H})$ & $C(\mu \mathrm{F})$ & $\begin{array}{c}T H D_{v}(\%) \\
\text { calculada }\end{array}$ & $\begin{array}{c}T H D_{v}(\%) \\
\text { medida }\end{array}$ \\
\hline 1 & 1 & 4,98 & 250 & 60 & 2,86 & 2,77 \\
\hline 2 & 1 & 4,98 & 500 & 60 & 1,43 & 1,64 \\
\hline 3 & 1 & 2,52 & 250 & 60 & 11,17 & 11,80 \\
\hline 4 & 1 & 2,52 & 500 & 60 & 5,58 & 5,03 \\
\hline 5 & 0,5 & 4,98 & 250 & 60 & 2,38 & 1,86 \\
\hline 6 & 0,5 & 4,98 & 500 & 60 & 1,19 & 1,21 \\
\hline 7 & 0,5 & 2,52 & 250 & 60 & 9,31 & 10,30 \\
\hline 8 & 0,5 & 2,52 & 500 & 60 & 4,65 & 5,45 \\
\hline
\end{tabular}

a experimentalmente as $T H D_{v}$ em vários testes, em malha aberta, para diferentes combinações de parâmetros $L, C$ e $f_{s}$. As $T H D_{v}$ obtidas experimentalmente nestes testes foram comparadas com os valores previstos, pela metodologia proposta, através da eq. (10). Os resultados, comparativos são apresentados na Tabela $\mathrm{V}$, e demonstram a validade da metodologia proposta.

\subsection{Determinação da freqüência natural do filtro para inversores trifásicos a quatro fios}

Este exemplo apresenta a descrição do procedimento para obtenção da freqüência natural de um de um filtro LC trifásico com saída a quatro fios. A representação da topologia do inversor está ilustrada na Fig. 11, sendo que os detalhes da modulação space vector utilizada estão mostrados no Apêndice A.2.

\subsubsection{Obtenção do índice $n D F_{2}(p, m)$}

As etapas para obtenção de $n D F_{2}(m)$, para esta modulação space vector, são as seguintes:

Etapa 1: Determinação do modelo do filtro do inversor.

$\left(\begin{array}{l}\left|V_{a o}[n]\right| \\ \left|V_{b o}[n]\right| \\ \left|V_{c o}[n]\right|\end{array}\right)=\left(\left.\begin{array}{lll}\left|G_{1}(s)\right| & \left|G_{2}(s)\right| & \left|G_{2}(s)\right| \\ \left|G_{2}(s)\right| & \left|G_{1}(s)\right| & \left|G_{2}(s)\right| \\ \left|G_{2}(s)\right| & \left|G_{2}(s)\right| & \left|G_{1}(s)\right|\end{array}\right|_{s=j \omega_{1} n}\left(\begin{array}{l}\mid U_{a o}[n] \\ \left|U_{b o}[n]\right| \\ \left|U_{c o}[n]\right|\end{array}\right)\right.$

$$
\begin{aligned}
G_{1}(s) & =\frac{3}{4} \frac{\omega_{r}{ }^{2}\left(s^{2}+2 \zeta \omega_{r} s+\omega_{r}{ }^{2} / 3\right)}{\left(s^{2}+2 \zeta \omega_{r} s+\omega_{r}{ }^{2}\right)\left(s^{2}+2 \zeta \omega_{r} s+\omega_{r}{ }^{2} / 4\right)} \\
G_{2}(s) & =-\frac{1}{4} \frac{\omega_{r}{ }^{2}\left(s^{2}+2 \zeta \omega_{r} s\right)}{\left(s^{2}+2 \zeta \omega_{r} s+\omega_{r}{ }^{2}\right)\left(s^{2}+2 \zeta \omega_{r} s+\omega_{r}{ }^{2} / 4\right)}
\end{aligned}
$$

$\omega_{r}=\frac{1}{\sqrt{L C}}, \quad \zeta=\frac{1}{2 R} \sqrt{\frac{L}{C}}$,

$\left[V_{1}[n] V_{2}[n] V_{3}[n]\right]^{\mathrm{T}}=\left[V_{a o}[n] V_{b o}[n] V_{c o}[n]\right]^{\mathrm{T}} \mathrm{e}$

$\left[U_{1}[n] U_{2}[n] V_{3}[n]\right]^{\mathrm{T}}=\left[\begin{array}{ll}U_{a o}[n] & U_{b o}[n] U_{c o}[n]\end{array}\right]^{\mathrm{T}}$.

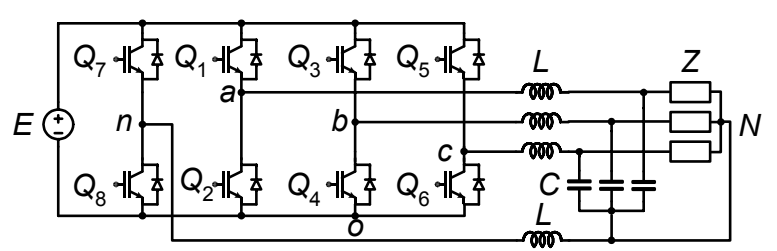

Figura 11. Inversor de tensão trifásico de quatro braços a quatro fios com filtro LC de segunda ordem. 
Etapa 2: Aproximação assintótica:

$\left|\mathbf{G}_{1,1}{ }^{*}\left(j \omega_{1} n\right)\right|\left|U_{1}[n]\right|=\left\{\begin{array}{c}\mid U_{1}[1] \quad, n=1 \\ \frac{3}{4}\left(\frac{f_{r}}{f_{1}}\right)^{2} \frac{1}{n^{2}}\left|U_{1}[n]\right|, n \gg \frac{f_{r}}{f_{1}}, n \in \mathbb{N}^{+}\end{array}\right.$

$\left|\mathbf{G}_{1,2}{ }^{*}\left(j \omega_{1} n\right)\right|\left|U_{2}[n]\right|=\left\{\begin{array}{cl}0 & , n=1 \\ -\frac{1}{4}\left(\frac{f_{r}}{f_{1}}\right)^{2} \frac{1}{n^{2}}\left|U_{2}[n]\right|, n \gg \frac{f_{r}}{f_{1}}, n \in \mathbb{N}^{+}\end{array}\right.$

Etapa 3: A análise da variação resistência de carga para $G_{1}(s)$ e $G_{2}(s)$ é mostrada na Fig. 12. Observa-se, nesta figura, que os erros associados às variações de carga consideradas são suficientemente pequenos para $\omega<0,1 \omega_{r} \mathrm{e}$ $\omega>5 \omega_{r}$. Note que mesmo sendo considerável a diferença das respostas para a faixa das baixas freqüências de $G_{2}(s)$, as amplitudes das componentes fundamentais destas tensões são insignificantes em relação à amplitude da fundamental de $G_{1}(s)$, que é de $0 \mathrm{~dB}$. Logo, a aproximação de $G_{2}(s)$ por zero nas baixas freqüências sem erros significativos é garantida.

Etapa 4: Determinação de $m$. Para este inversor, a máxima amplitude da senóide de saída possível de ser gerada, entre fase e neutro, sem sobremodulação é igual a $E / \sqrt{3}, \operatorname{logo}$ $\sqrt{3}\left|U_{x}[1]\right| / E$.

Etapa 5: Verificação da adequação da estratégia de modulação com as hipóteses assumidas. Realizando-se a transformada rápida de Fourier de $u_{a n}, u_{b n}$ e $u_{c n}$ obtidas a

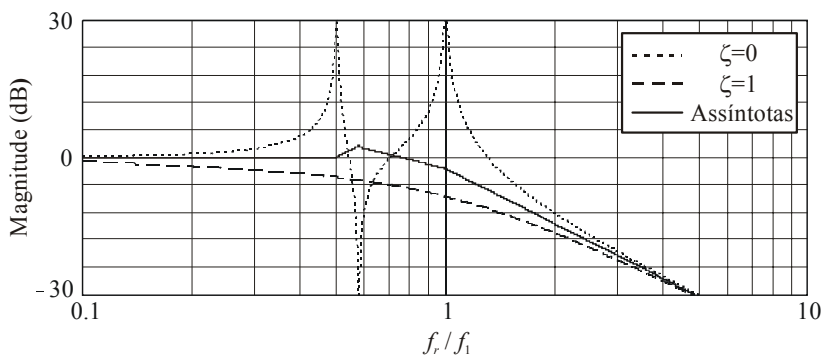

(a)

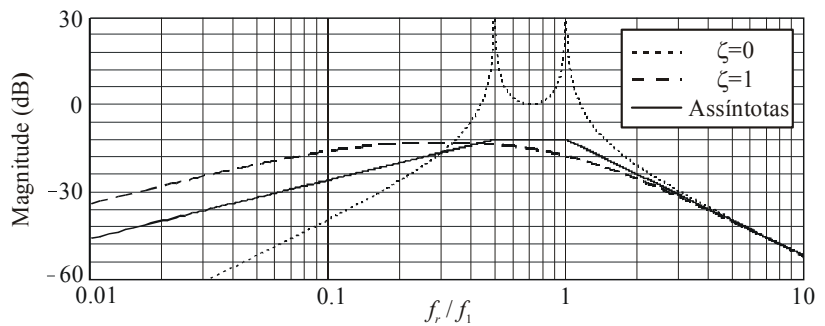

(b)

Figura 12. Comparação entre a aproximação assintótica e a respostas em freqüência para o filtro mostrado na Fig.6(b).
(a) $G_{1}(s)$
(b) $G_{2}(s)$.

partir de uma senóide de freqüência $f_{1}$ modulada com uma freqüência $f_{s}$, chega-se a um espectro com a forma similar à apresentada na Fig. 3, no qual se verifica que esta resposta espectral atende às hipóteses 7,8 e 9 assumidas.

Etapa 6: Traçado da curva de $n D F_{2}(p, m)$. Esta curva é obtida a partir de (18), sendo $a_{1}=m_{s}, a_{2}=2 m_{s}, a_{3}=3 m_{s}$, e $b_{1}=5$, $b_{2}=10, b_{3}=15$. Os grupos de harmônicas de freqüências maior que estas podem ser desconsiderados, uma vez que estas harmônicas são sensivelmente atenuadas pelo filtro. Com esta informação do espetro de freqüências da estratégia de modulação utilizada, chega-se a curva para o fator $n D F_{2}(p, m)$, mostrada na curva da Fig. 14 para inversor trifásico a quatro fios. Como os sinais gerados pelo inversor são simétricos, $n D F_{2}(p, m)=n D F_{2}(m)$.

\subsubsection{Resultados experimentais}

Neste exemplo, estipulou-se as seguintes especificações para o projeto do filtro: $T H D_{v}=3 \%, f_{1}=60 \mathrm{~Hz}, f_{s}=4,98 \mathrm{kHz}$, $m=1$.

A determinação da freqüência natural do filtro então é efetuada usando (14), onde $m_{s}=f_{s} / f_{1}=83$ e o fator de distorção $n D F_{2}=0,45$ é obtido na Fig.14 para $m=1$. Aplicando-se estes dados em (14), obteve-se a freqüência natural de $f_{r}=1285 \mathrm{~Hz}$. Para verificação experimental do procedimento de projeto foi implementado o inversor com o filtro projetado. Escolheu-se para a implementação um indutor de $L=250 \mu \mathrm{H}$, sendo o capacitor determinado pela relação $\omega_{r}{ }^{2}=1 / L C$, o que resultou em $C=61,82 \mu \mathrm{F}$, sendo utilizado $C=60 \mu \mathrm{F}$, que é a capacitância mais próxima comercialmente existente. A $T H D_{v}$ obtida experimentalmente, com o inversor operando sem carga, foi de 2,56\%. As Fig.13(a) e Fig.13(b) mostram, respectivamente, o padrão space vector utilizado e a tensão entre as fases de saída para esta topologia.

De maneira similar à descrita na seção 5.1 .3 e 5.2.2, determinou-se, experimentalmente, a $T H D_{v}$ em vários testes em malha aberta, para diferentes combinações de parâmetros $L, C$ e $f_{s}$. As $T H D_{v}$ obtidas experimentalmente nestes testes foram comparadas com os valores previstos, pela metodologia proposta, através da eq. (10). Os resultados, comparativos são apresentados na Tabela VI, e demonstram a validade da metodologia proposta.

\section{CONCLUSÃO}

Este artigo apresenta um procedimento sistematizado de projeto de filtros de segunda ordem para inversores de tensão com modulação PWM digital, sendo aplicável tanto para inversores que alimentam cargas lineares quanto nãolineares. $\mathrm{O}$ procedimento apresentado apresenta contribuições na metodologia para determinação da freqüência natural do filtro e no procedimento para a obtenção da relação entre as capacitâncias e indutâncias do 

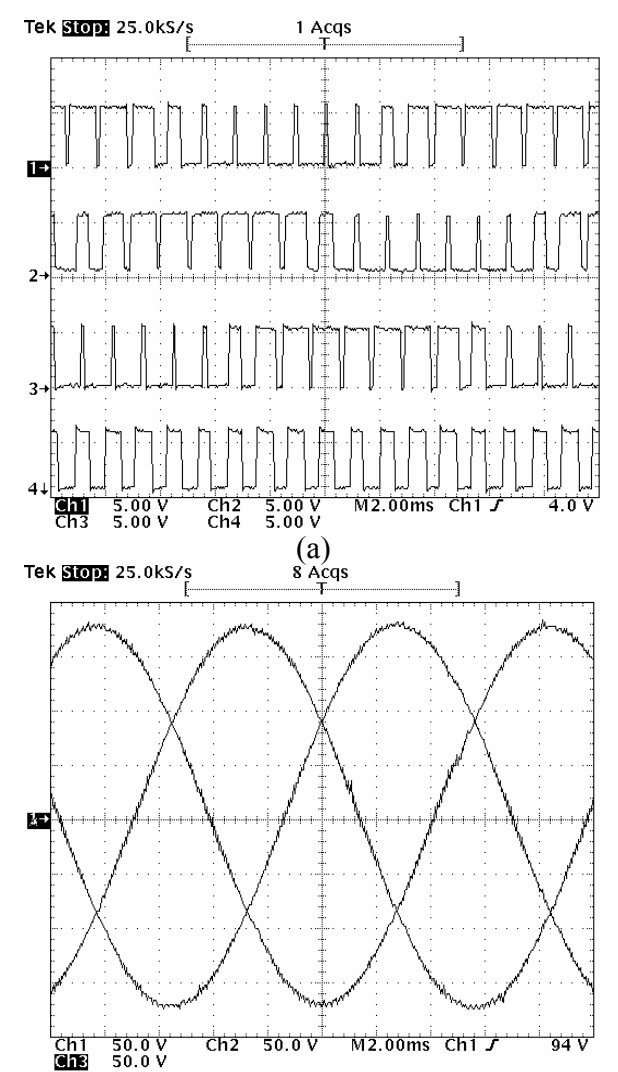

(b)

Figura 13. Formas de onda experimentais obtidas para o inversor trifásico a quatro fios com estratégia de modulação space vector com o filtro de segunda-ordem projetado na Seção 5.3.2.

(a) Padrão PWM gerado pelo DSP (5V/div, $2 \mathrm{~ms} / \mathrm{div})$.

(b) Tensão $v_{a n}$ na saída do filtro (50V/div, $\left.2 \mathrm{~ms} / \mathrm{div}\right)$.

mesmo. Os exemplos demonstram que a utilização da metodologia apresentada é direta e que a mesma pode ser aplicada para a maioria dos inversores de tensão alimentados em tensão com modulação PWM digital, desde que as topologias dos filtros e as estratégias de modulação respeitem as hipóteses assumidas. Os resultados de avaliações experimentais, realizadas para três topologias distintas de inversores, demonstram a validade da metodologia proposta.

TABELA VI - COMPARAÇÕES ENTRE A THD CALCULADA E OBTIDA EXPERIMENTALMENTE PARA O INVERSOR TRIFÁSICO COM QUATRO BRAÇOS A QUATROS FIOS COM MODULAÇÃO SPACE VECTOR

\begin{tabular}{|c|c|c|c|c|c|c|}
\hline $\begin{array}{l}\text { Número } \\
\text { do teste }\end{array}$ & $m$ & $f_{\mathrm{s}}(\mathrm{kHz})$ & $L(\mu \mathrm{H})$ & $C(\mu \mathrm{F})$ & $\begin{array}{l}T H D_{v}(\%) \\
\text { calculada }\end{array}$ & $\begin{array}{c}T H D_{v}(\%) \\
\text { medida }\end{array}$ \\
\hline 1 & 1 & 4,98 & 250 & 60 & 3,06 & 2,56 \\
\hline 2 & 1 & 4,98 & 500 & 60 & 1,53 & 1,37 \\
\hline 3 & 1 & 2,52 & 250 & 60 & 11,67 & 13,79 \\
\hline 4 & 1 & 2,52 & 500 & 60 & 5,98 & 6,31 \\
\hline 5 & 0,5 & 4,98 & 250 & 60 & 2,52 & 2,38 \\
\hline 6 & 0,5 & 4,98 & 500 & 60 & 1,29 & 1,48 \\
\hline 7 & 0,5 & 2,52 & 250 & 60 & 9,84 & 8,28 \\
\hline 8 & 0,5 & 2,52 & 500 & 60 & 4,92 & 5,33 \\
\hline
\end{tabular}

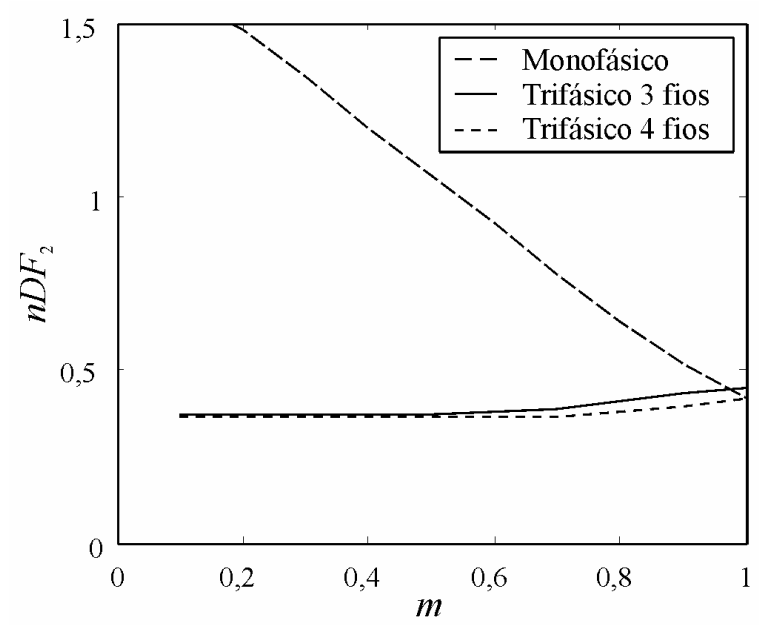

Figura 14. Curvas para taxa de distorção de segunda ordem $n D F_{2}(m)$ para uma estratégia de modulação space vector monofásica, trifásica a três fios e trifásica a quatro fios.

\section{REFERÊNCIAS BIBLIOGRÁFICAS}

Boost, M.A. e Ziogas, P.D. (1988). State-of-the-Art Carrier PWM Techniques: A Critical Evaluation. IEEE Trans. Ind. Applicat., 24(2): 271-280.

Botterón, F., Pinheiro, H, Gründling, H.A., Pinheiro, J.R. e Hey, H.L. (2001). Digital Voltage and Current Controllers for Three-Phase PWM Inverter for UPS Applications. Conf. IEEE IAS'01 Annual Meet., pp. 2667-2674.

Bowes, S. R., (1995). Advanced Regular-Sampled PWM Control Techniques for Drives and Static Power Converters. IEEE Trans. Ind. Electron., 42(4):367373.

Bowes, S. R. e Lai, Y. S. (1997). The Relationship Between Space-Vector Modulation and Regular-Sampled PWM. IEEE Trans. Ind. Electron., 44(5): 670-679.

Dahono, P. A., Purwadi, A. e Qamaruzzaman (1995). An LC Filter Design Method for Single-Phase PWM Inverters. Proc. IEEE PEDS'95, v. 2, pp. 571-576.

Dewan, S.B. e Ziogas, P.D. (1979). Optimum Filter Design for a Single Phase Solid-State UPS System. IEEE Trans. Ind. Applicat., IA-15(6): 664-669.

Enjeti, P. N., Ziogas, P. D. e Lindsay, J. F. (1990). Programmed PWM Techniques to Eliminate Harmonics: A Critical Evaluation. IEEE Trans. Ind. Applicat., 26(2): 302-316.

Holtz, J. (1992). Pulsewidth Modulation - A Survey, IEEE Trans. Ind. Electr., 39(6): 410-419. 
IEC 62040-3 (1999). Uninterruptible power systems (UPS) - Part 3: Method of specifying the performance and test requirements. International Electrotechnical Commission, Genebra, Suíça.

Kim, J., Choi J. e Hong H. (2000). Output LC Filter Design on Voltage Source Inverter Considering the Performance of the Controller. Proc. PowerCon 2000, v. 3, pp. 1659-1664.

Kusko, A., Galler, D. e Medora, N. (1990). Output Impedance of PWM UPS Inverter-Feedback vs. Filters. Conf. Rec. of the 1990 IEEE IAS Annual Meeting, v.2, pp.1044-1048.

Mansoor, A., Grady, W. M., Thallam, R. S., Doyle, M. T., Krein, S. D., Samotyj, M. J. (1995). Effect of Supply Voltage Harmonics on the Input Current of SinglePhase Diode Bridge Rectifier Loads. IEEE Trans. on Power Delivery, 10(3): 1416-1422.

Michels, L., Camargo, R. F., Marques, J., Botterón, F., Gonzatti, F. e Pinheiro, H. (2003). Simple Filter Design Procedure for Voltage Fed Space Vector Modulated Converters. Anais do $7^{\circ}$ Congresso Brasileiro de Eletrônica de Potência, pp. 430-437.

Mohan, N., Undeland, T. M. e Robbins, W. P. (1995). Power Electronics: Converts, Applications and Design. John Wiley \& Sons Inc, $3^{\text {a }}$ edição, New York, NY, EUA.

Patel, H. S. e Hoft, R. G. (1973). Generalized Techniques of Harmonic Elimination and Voltage Control in Thyristor Inverters: Part I - Harmonic Elimination. IEEE Trans. Ind. Applicat., 9(3): 110-117.

Pinheiro, H, Botterón, F., Rech, C., Schuch, L., Camargo, R. F., Hey, H. L., Gründling, H. A. e Pinheiro, J. R. (2002). Space Vector Modulation for VoltageSource Inverters: A Unified Approach. IEEE $28^{\text {th }}$ IECON Conf. Proc., v.1, pp. 23-29.

Redl, R. (1996). Power Electronics and Electromagnetic Compatibility. IEEE $27^{\text {th }}$ PESC Conf. Rec., v.1, pp. 15-21.

Ryu, B., Kim, J., Choi J. e Choi C. (2002). Design and Analysis of Output Filter for 3-phase UPS Inverter; Proc of the IEEE PCC 2002, v.3, pp. 941-946.

Vukosavic, S. et al. (1990). Reduction of the output impedance of PWM inverters for uninterruptible power supplies. IEEE $21^{\text {th }}$ PESC Conf. Rec., pp. $757-762$

van der Broeck, H. W., Skudelny, H. C. e Stanke, G. V. (1988). Analysis and realization of a pulsewidth modulator based on voltage space vectors, IEEE Trans. Ind. Applicat., 24(1): 142-150.

van der Broeck e Miller, M. (1995). Harmonics in DC to AC converters of single phase uninterruptible power supplies, Proc. of the IEEE $17^{\text {th }}$ INTELEC, v. 1, pp. 653-658.

\section{APÊNDICE}

\section{A.1 Modulação space vector empregada na Seção 5.2}

O inversor apresentado na Fig. 9 apresenta oito possíveis vetores, que estão descritos na Tabela VII. A Fig. 15 apresenta o espaço das tensões de saída transformado de abc para $\alpha \beta$, sendo esta transformação dada por:

$$
\mathbf{T}_{\alpha \beta}=\sqrt{\frac{2}{3}}\left[\begin{array}{ccc}
1 & -1 / 2 & -1 / 2 \\
0 & \sqrt{3} / 2 & -\sqrt{3} / 2
\end{array}\right]
$$

Como mostrado na Fig. 15, o espaço das tensões de saída em coordenadas $\alpha \beta$ possui o formato hexagonal, possuindo seis regiões distintas, de formato triangular, contendo cada um deles quatro vetores. A Tabela VIII mostra os vetores que formam cada um destas regiões triangulares, além das condições de pertinência e as condições-limite para os vetores de modulação. As retas de separação entre as regiões triângulares e as retas limites do espaço das tensões de saída empregadas na Tabela VIII são dadas a seguir, além da seqüência de comutação que foi empregada em cada região triangular para o exemplo apresentado.

Retas de separação entre as regiões triangulares:

$\mathrm{R}_{1} \therefore u_{\beta}+\sqrt{3} u_{\alpha}=0, \mathrm{R}_{2} \therefore u_{\beta}-\sqrt{3} \quad u_{\alpha}=0, \mathrm{R}_{3} \therefore u_{\beta}=0$

Retas limite do espaço das tensões de saída:

$\mathrm{R}_{4} \therefore u_{\beta}+\sqrt{3} \quad u_{\alpha}-\sqrt{2}=0, \mathrm{R}_{5} \therefore u_{\beta}-\sqrt{2} / 2=0$,

$\mathrm{R}_{6} \therefore u_{\beta}-\sqrt{3} \quad u_{\alpha}-\sqrt{2}=0 \quad \mathrm{R}_{7} \therefore u_{\beta}+\sqrt{3} \quad u_{\alpha}+\sqrt{2}=0$,

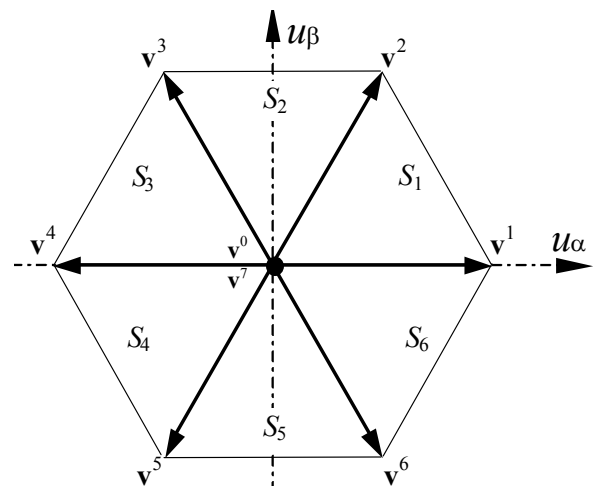

Figura 15. Espaço das tensões de saída dos inversores trifásicos a três fios com três braços em coordenadas $\alpha \beta$. 
TABELA VII. POSSÍVEIS VETORES DE COMUTAÇÃO DOS INVERSORES TRIFÁSICOS A TRÊS FIOS COM TRÊS BRAÇOS

\begin{tabular}{ccccccccc}
\hline$Q_{1}$ & $Q_{3}$ & $Q_{5}$ & $v_{a b}^{\prime}$ & $v_{b c}^{\prime}$ & $v_{c a}^{\prime}$ & $v_{\alpha}^{\prime}$ & $v_{\beta}^{\prime}$ & Vetores \\
\hline 0 & 0 & 0 & 0 & 0 & 0 & 0 & 0 & $\mathbf{v}^{0}$ \\
\hline 0 & 0 & 1 & 0 & -1 & 1 & $\sqrt{2 / 3}$ & 0 & $\mathbf{v}^{1}$ \\
\hline 0 & 1 & 0 & -1 & 1 & 0 & $1 / \sqrt{6}$ & $1 / \sqrt{2}$ & $\mathbf{v}^{2}$ \\
\hline 0 & 1 & 1 & -1 & 0 & 1 & $-1 / \sqrt{6}$ & $1 / \sqrt{2}$ & $\mathbf{v}^{3}$ \\
\hline 1 & 0 & 0 & 1 & 0 & -1 & $-\sqrt{2 / 3}$ & 0 & $\mathbf{v}^{4}$ \\
\hline 1 & 0 & 1 & 1 & -1 & 0 & $-1 / \sqrt{6}$ & $-1 / \sqrt{2}$ & $\mathbf{v}^{5}$ \\
\hline 1 & 1 & 0 & 0 & 1 & -1 & $1 / \sqrt{6}$ & $-1 / \sqrt{2}$ & $\mathbf{v}^{6}$ \\
\hline 1 & 1 & 1 & 0 & 0 & 0 & 0 & 0 & $\mathbf{v}^{7}$ \\
\hline
\end{tabular}

TABELA VIII. VETORES DE COMUTAÇÃO, CONDIÇÕES DE SEPARAÇÃO E CONDICÕES LIMITES DE CADA SETOR DOS INVERSORES TRIFÁSICOS A TRÊS FIOS COM TRÊS BRAÇOS

\begin{tabular}{cccc}
\hline Setor & $\begin{array}{c}\text { Vetores de } \\
\text { comutação do setor }\end{array}$ & $\begin{array}{c}\text { Condições de } \\
\text { pertinência }\end{array}$ & $\begin{array}{c}\text { Condições- } \\
\text { limite }\end{array}$ \\
\hline 1 & $\mathbf{v}^{0}, \mathbf{v}^{1}, \mathbf{v}^{2}, \mathbf{v}^{7}$ & $\left(R_{1} ; R_{3}\right)>0 ; R_{2} \leq 0$ & $R_{4}<1$ \\
\hline 2 & $\mathbf{v}^{0}, \mathbf{v}^{2}, \mathbf{v}^{3}, \mathbf{v}^{7}$ & $\left(R_{1} ; R_{2} ; R_{3}\right)>0$ & $R_{5}<1$ \\
\hline 3 & $\mathbf{v}^{0}, \mathbf{v}^{3}, \mathbf{v}^{4}, \mathbf{v}^{7}$ & $\left(R_{2} ; R_{3}\right)>0 ; R_{1} \leq 0$ & $R_{6}<1$ \\
\hline 4 & $\mathbf{v}^{0}, \mathbf{v}^{4}, \mathbf{v}^{5}, \mathbf{v}^{7}$ & $R_{2}>0 ;\left(R_{1} ; R_{3}\right) \leq 0$ & $R_{7}<1$ \\
\hline 5 & $\mathbf{v}^{0}, \mathbf{v}^{5}, \mathbf{v}^{6}, \mathbf{v}^{7}$ & $\left(R_{1} ; R_{2} ; R_{3}\right) \leq 0$ & $R_{8}<1$ \\
\hline 6 & $\mathbf{v}^{0}, \mathbf{v}^{1}, \mathbf{v}^{6}, \mathbf{v}^{7}$ & $R_{1}>0 ;\left(R_{2} ; R_{3}\right) \leq 0$ & $R_{9}<1$ \\
\hline
\end{tabular}

$\mathrm{R}_{8} \therefore u_{\beta}+\sqrt{2} / 2=0, \mathrm{R}_{9} \therefore u_{\beta}-\sqrt{3} \quad u_{\alpha}+\sqrt{2}=0$

Seqüencia de comutação em cada região triangular:

$S_{1}: \mathbf{v}^{0}-v^{1}-v^{2}-v^{7}-v^{2}-v^{1}-v^{0} ; S_{2}: \mathbf{v}^{0}-v^{3}-v^{2}-v^{7}-v^{2}-v^{3}-v^{0} ;$

$S_{3}: v^{0}-v^{3}-v^{4}-v^{7}-v^{4}-v^{3}-v^{0} ; S_{4}: v^{0}-v^{5}-v^{4}-v^{7}-v^{4}-v^{5}-v^{0} ;$

$S_{5}: \mathbf{v}^{0}-\mathbf{v}^{5}-\mathbf{v}^{6}-\mathbf{v}^{7}-\mathbf{v}^{6}-\mathbf{v}^{5}-\mathbf{v}^{0} ; S_{6}: \mathbf{v}^{0}-\mathbf{v}^{1}-\mathbf{v}^{6}-\mathbf{v}^{7}-\mathbf{v}^{6}-\mathbf{v}^{1}-\mathbf{v}^{0}$

\section{A.2 Modulação space vector empregada na Seção 5.3}

O inversor apresentado na Fig. 11 apresenta dezesseis possíveis vetores, que estão descritos na Tabela IX. A Fig.16 apresenta o espaço das tensões de saída transformado de abc para $0 \alpha \beta$, sendo esta transformação dada por:

$$
\mathbf{T}_{0 \alpha \beta}=\sqrt{\frac{2}{3}}\left[\begin{array}{ccc}
1 / \sqrt{2} & 1 / \sqrt{2} & 1 / \sqrt{2} \\
1 & -1 / 2 & -1 / 2 \\
0 & \sqrt{3} / 2 & -\sqrt{3} / 2
\end{array}\right]
$$

Como mostrado na Fig. 16, o espaço das tensões de saída em coordenadas $0 \alpha \beta$ possui o formato dodecaédrico, possuindo vinte e quatro regiões distintas, de formato tetraédrico, contendo cada um deles quatro vetores. A Tabela $\mathrm{X}$ mostra os vetores que formam cada um destes tetraedros, além das condições de pertinência e as condições-limite para os vetores de referência. Os planos de separação entre os tetraedros e os planos limites do espaço das tensões de saída empregadas na Tabela IX são dadas a seguir, além da seqüência de comutação que foi empregada em cada região triangular para o exemplo apresentado.

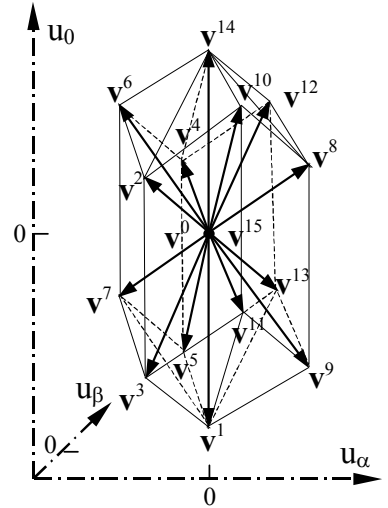

Figura 16. Espaço das tensões de saída dos inversores trifásicos a quatro fios com quatro braços em coordenadas $0 \alpha \beta$.

Planos de separação entre os tetraedros:

$\mathrm{P}_{1} \therefore \sqrt{2} u_{\beta}=0$;

$\mathrm{P}_{2} \therefore-\sqrt{6} / 2 u_{\alpha}+\sqrt{2} / 2 u_{\beta}=0$;

$\mathrm{P}_{3} \therefore \sqrt{6} / 2 u_{\alpha}+\sqrt{2} / 2 u_{\beta}=0$;

$\mathrm{P}_{4} \therefore \sqrt{6} / 3 u_{\alpha}+\sqrt{3} / 3 u_{0}=0$;

$\mathrm{P}_{5} \therefore-\sqrt{6} / 6 u_{\alpha}-\sqrt{2} / 2 u_{\beta}+\sqrt{3} / 3 u_{0}=0 ;$

$\mathrm{P}_{6} \therefore-\sqrt{6} / 6 u_{\alpha}+\sqrt{2} / 2 u_{\beta}+\sqrt{3} / 3 u_{0}=0$

Planos limite do espaço das tensões de saída:

$\mathrm{P}_{7} \therefore \sqrt{3} / 2 u_{\alpha}+\sqrt{3} / 3 u_{0}=1$;

$\mathrm{P}_{8} \therefore-\sqrt{6} / 6 u_{\alpha}+\sqrt{2} / 2 u_{\beta}+\sqrt{3} / 3 u_{0}=1 ;$

$\mathrm{P}_{9} \therefore-\sqrt{6} / 6 u_{\alpha}-\sqrt{2} / 2 u_{\beta}+\sqrt{3} / 3 u_{0}==1$;

$\mathrm{P}_{10} \therefore \sqrt{6} / 2 u_{\alpha}+\sqrt{2} / 2 u_{\beta}=1$;

$\mathrm{P}_{11} \therefore \sqrt{2} u_{\beta}=1$;

TABELA IX. POSSÍVEIS VETORES DE COMUTAÇ̃̃O DOS INVERSORES TRIFÁSICOS A QUATRO FIOS COM QUATRO BRAÇOS

\begin{tabular}{ccccccccccc}
\hline$Q_{1}$ & $Q_{3}$ & $Q_{5}$ & $Q_{7}$ & $v_{a n}^{\prime}$ & $v_{b n}^{\prime}$ & $v_{c n}^{\prime}$ & $v_{0}^{\prime}$ & $v_{\alpha}^{\prime}$ & $v_{\beta}^{\prime}$ & Vectors \\
\hline 0 & 0 & 0 & 0 & 0 & 0 & 0 & 0 & 0 & 0 & $\mathbf{v}^{0}$ \\
\hline 0 & 0 & 0 & 1 & -1 & -1 & -1 & 0 & 0 & $-\sqrt{3}$ & $\mathbf{v}^{1}$ \\
\hline 0 & 0 & 1 & 0 & 0 & 0 & 1 & $-\sqrt{6} / 6$ & $-\sqrt{2} / 2$ & $\sqrt{3} / 3$ & $\mathbf{v}^{2}$ \\
\hline 0 & 0 & 1 & 1 & -1 & -1 & 0 & $-\sqrt{6} / 6$ & $-\sqrt{2} / 2$ & $-2 \sqrt{3} / 3$ & $\mathbf{v}^{3}$ \\
\hline 0 & 1 & 0 & 0 & 0 & 1 & 0 & $-\sqrt{6} / 6$ & $\sqrt{2} / 2$ & $\sqrt{3} / 3$ & $\mathbf{v}^{4}$ \\
\hline 0 & 1 & 0 & 1 & -1 & 0 & -1 & $-\sqrt{6} / 6$ & $\sqrt{2} / 2$ & $-2 \sqrt{3} / 3$ & $\mathbf{v}^{5}$ \\
\hline 0 & 1 & 1 & 0 & 0 & 1 & 1 & $-\sqrt{6} / 3$ & 0 & $2 \sqrt{3} / 3$ & $\mathbf{v}^{6}$ \\
\hline 0 & 1 & 1 & 1 & -1 & 0 & 0 & $-\sqrt{6} / 3$ & 0 & $-\sqrt{3} / 3$ & $\mathbf{v}^{7}$ \\
\hline 1 & 0 & 0 & 0 & 1 & 0 & 0 & $\sqrt{6} / 3$ & 0 & $\sqrt{3} / 3$ & $\mathbf{v}^{8}$ \\
\hline 1 & 0 & 0 & 1 & 0 & -1 & -1 & $\sqrt{6} / 3$ & 0 & $-2 \sqrt{3} / 3$ & $\mathbf{v}^{9}$ \\
\hline 1 & 0 & 1 & 0 & 1 & 0 & 1 & $\sqrt{6} / 6$ & $-\sqrt{2} / 2$ & $2 \sqrt{3} / 3$ & $\mathbf{v}^{10}$ \\
\hline 1 & 0 & 1 & 1 & 0 & -1 & 0 & $\sqrt{6} / 6$ & $-\sqrt{2} / 2$ & $-\sqrt{3} / 3$ & $\mathbf{v}^{11}$ \\
\hline 1 & 1 & 0 & 0 & 1 & 1 & 0 & $\sqrt{6} / 6$ & $\sqrt{2} / 2$ & $2 \sqrt{3} / 3$ & $\mathbf{v}^{12}$ \\
\hline 1 & 1 & 0 & 1 & 0 & 0 & -1 & $\sqrt{6} / 6$ & $\sqrt{2} / 2$ & $\sqrt{3} / 3$ & $\mathbf{v}^{13}$ \\
\hline 1 & 1 & 1 & 0 & 1 & 1 & 1 & 0 & 0 & $\sqrt{3}$ & $\mathbf{v}^{14}$ \\
\hline 1 & 1 & 1 & 1 & 0 & 0 & 0 & 0 & 0 & 0 & $\mathbf{v}^{15}$ \\
\hline & & & & & & & & &
\end{tabular}




$$
\begin{aligned}
& \mathrm{P}_{12} \therefore-\sqrt{6} / 2 u_{\alpha}+\sqrt{2} / 2 u_{\beta}=1 ; \\
& \mathrm{P}_{13} \therefore-\sqrt{6} / 2 u_{\alpha}-\sqrt{2} / 2 u_{\beta}=1 ; \\
& \mathrm{P}_{14} \therefore-\sqrt{2} u_{\beta}=1 ; \\
& \mathrm{P}_{15} \therefore \sqrt{6} / 2 u_{\alpha}-\sqrt{2} / 2 u_{\beta}=1 ; \\
& \mathrm{P}_{16} \therefore \sqrt{6} / 6 u_{\alpha}+\sqrt{2} / 2 u_{\beta}-\sqrt{3} / 3 u_{0}=1 ;
\end{aligned}
$$

Seqüencia de comutação em cada tetraedro:

$S_{1}: \mathbf{v}^{0}-\mathbf{v}^{8}-\mathbf{v}^{12}-\mathbf{v}^{14}-\mathbf{v}^{15}-\mathbf{v}^{14}-\mathbf{v}^{12}-\mathbf{v}^{8}-\mathbf{v}^{0}$;

$S_{2}: \mathbf{v}^{0}-\mathbf{v}^{8}-\mathbf{v}^{12}-\mathbf{v}^{13}-\mathbf{v}^{15}-\mathbf{v}^{13}-\mathbf{v}^{12}-\mathbf{v}^{8}-\mathbf{v}^{0}$;

$S_{3}: \mathbf{v}^{0}-\mathbf{v}^{8}-\mathbf{v}^{9}-\mathbf{v}^{13}-\mathbf{v}^{15}-\mathbf{v}^{13}-\mathbf{v}^{9}-\mathbf{v}^{8}-\mathbf{v}^{0}$;

$S_{4}: \mathbf{v}^{0}-\mathbf{v}^{1}-\mathbf{v}^{9}-\mathbf{v}^{13}-\mathbf{v}^{15}-\mathbf{v}^{13}-\mathbf{v}^{9}-\mathbf{v}^{1}-\mathbf{v}^{0}$;

$S_{5}: \mathbf{v}^{0}-\mathbf{v}^{4}-\mathbf{v}^{12}-\mathbf{v}^{14}-\mathbf{v}^{15}-\mathbf{v}^{14}-\mathbf{v}^{12}-\mathbf{v}^{4}-\mathbf{v}^{0}$

$S_{6}: \mathbf{v}^{0}-\mathbf{v}^{4}-\mathbf{v}^{12}-\mathbf{v}^{13}-\mathbf{v}^{15}-\mathbf{v}^{13}-\mathbf{v}^{12}-\mathbf{v}^{4}-\mathbf{v}^{0}$

$S_{7}: \mathbf{v}^{0}-\mathbf{v}^{4}-\mathbf{v}^{5}-\mathbf{v}^{13}-\mathbf{v}^{15}-\mathbf{v}^{13}-\mathbf{v}^{5}-\mathbf{v}^{4}-\mathbf{v}^{0}$

$S_{8}: \mathbf{v}^{0}-\mathbf{v}^{1}-\mathbf{v}^{5}-\mathbf{v}^{13}-\mathbf{v}^{15}-\mathbf{v}^{13}-\mathbf{v}^{5}-\mathbf{v}^{1}-\mathbf{v}^{0}$;

$S_{9}: \mathbf{v}^{0}-\mathbf{v}^{4}-\mathbf{v}^{6}-\mathbf{v}^{14}-\mathbf{v}^{15}-\mathbf{v}^{14}-\mathbf{v}^{6}-\mathbf{v}^{4}-\mathbf{v}^{0}$;

$S_{10}: \mathbf{v}^{0}-\mathbf{v}^{4}-\mathbf{v}^{6}-\mathbf{v}^{7}-\mathbf{v}^{15}-\mathbf{v}^{7}-\mathbf{v}^{6}-\mathbf{v}^{4}-\mathbf{v}^{0} ;$

$S_{11}: \mathbf{v}^{0}-\mathbf{v}^{4}-\mathbf{v}^{5}-\mathbf{v}^{7}-\mathbf{v}^{15}-\mathbf{v}^{7}-\mathbf{v}^{5}-\mathbf{v}^{4}-\mathbf{v}^{0}$

$S_{12}: \mathbf{v}^{0}-\mathbf{v}^{1}-\mathbf{v}^{5}-\mathbf{v}^{7}-\mathbf{v}^{15}-\mathbf{v}^{7}-\mathbf{v}^{5}-\mathbf{v}^{1}-\mathbf{v}^{0}$;

$S_{13}: \mathbf{v}^{0}-\mathbf{v}^{2}-\mathbf{v}^{6}-\mathbf{v}^{14}-\mathbf{v}^{15}-\mathbf{v}^{14}-\mathbf{v}^{6}-\mathbf{v}^{2}-\mathbf{v}^{0}$;

$S_{14}: \mathbf{v}^{0}-\mathbf{v}^{2}-\mathbf{v}^{6}-\mathbf{v}^{7}-\mathbf{v}^{15}-\mathbf{v}^{7}-\mathbf{v}^{6}-\mathbf{v}^{2}-\mathbf{v}^{0}$;

$S_{15}: \mathbf{v}^{0}-\mathbf{v}^{2}-\mathbf{v}^{3}-\mathbf{v}^{7}-\mathbf{v}^{15}-\mathbf{v}^{7}-\mathbf{v}^{3}-\mathbf{v}^{2}-\mathbf{v}^{0}$;

$S_{16}: \mathbf{v}^{0}-\mathbf{v}^{1}-\mathbf{v}^{3}-\mathbf{v}^{7}-\mathbf{v}^{15}-\mathbf{v}^{7}-\mathbf{v}^{3}-\mathbf{v}^{1}-\mathbf{v}^{0}$;

$S_{17}: \mathbf{v}^{0}-\mathbf{v}^{2}-\mathbf{v}^{10}-\mathbf{v}^{14}-\mathbf{v}^{15}-\mathbf{v}^{14}-\mathbf{v}^{10}-\mathbf{v}^{2}-\mathbf{v}^{0}$

$S_{18}: \mathbf{v}^{0}-\mathbf{v}^{2}-\mathbf{v}^{10}-\mathbf{v}^{11}-\mathbf{v}^{15}-\mathbf{v}^{11}-\mathbf{v}^{10}-\mathbf{v}^{2}-\mathbf{v}^{0}$;

$S_{19}: \mathbf{v}^{0}-\mathbf{v}^{2}-\mathbf{v}^{3}-\mathbf{v}^{11}-\mathbf{v}^{15}-\mathbf{v}^{11}-\mathbf{v}^{3}-\mathbf{v}^{2}-\mathbf{v}^{0}$;

$S_{20}: \mathbf{v}^{0}-\mathbf{v}^{1}-\mathbf{v}^{3}-\mathbf{v}^{11}-\mathbf{v}^{15}-\mathbf{v}^{11}-\mathbf{v}^{3}-\mathbf{v}^{1}-\mathbf{v}^{0}$;

$S_{21}: \mathbf{v}^{0}-\mathbf{v}^{8}-\mathbf{v}^{10}-\mathbf{v}^{14}-\mathbf{v}^{15}-\mathbf{v}^{14}-\mathbf{v}^{10}-\mathbf{v}^{8}-\mathbf{v}^{0}$;

$S_{22}: \mathbf{v}^{0}-\mathbf{v}^{8}-\mathbf{v}^{10}-\mathbf{v}^{11}-\mathbf{v}^{15}-\mathbf{v}^{11}-\mathbf{v}^{10}-\mathbf{v}^{8}-\mathbf{v}^{0}$;

$S_{23}: \mathbf{v}^{0}-\mathbf{v}^{8}-\mathbf{v}^{9}-\mathbf{v}^{11}-\mathbf{v}^{15}-\mathbf{v}^{11}-\mathbf{v}^{9}-\mathbf{v}^{8}-\mathbf{v}^{0}$;

\begin{tabular}{|c|c|c|c|}
\hline Tetraedro & $\begin{array}{c}\text { Vetores de } \\
\text { comutação do } \\
\text { tetraedro }\end{array}$ & $\begin{array}{l}\text { Condições de } \\
\text { pertinência }\end{array}$ & $\begin{array}{c}\text { Condições- } \\
\text { limite }\end{array}$ \\
\hline 1 & $\mathbf{v}^{0}, \mathbf{v}^{8}, \mathbf{v}^{12}, \mathbf{v}^{14}, \mathbf{v}^{15}$ & $\left(P_{1} ; P_{5}\right)>0 ; P_{2} \leq 0$ & $P_{7}=1$ \\
\hline 2 & $\mathbf{v}^{0}, \mathbf{v}^{8}, \mathbf{v}^{12}, \mathbf{v}^{13}, \mathbf{v}^{15}$ & $P_{6}>0 ;\left(P_{2} ; P_{5}\right) \leq 0$ & $P_{10}=1$ \\
\hline 3 & $\mathbf{v}^{0}, \mathbf{v}^{8}, \mathbf{v}^{9}, \mathbf{v}^{13}, \mathbf{v}^{15}$ & $\left(P_{1} ; P_{4}\right)>0 ; P_{6} \leq 0$ & $P_{10}=1$ \\
\hline 4 & $\mathbf{v}^{0}, \mathbf{v}^{1}, \mathbf{v}^{9}, \mathbf{v}^{13}, \mathbf{v}^{15}$ & $P_{1}>0 ;\left(P_{2} ; P_{4}\right) \leq 0$ & $P_{16}=1$ \\
\hline 5 & $\mathbf{v}^{0}, \mathbf{v}^{4}, \mathbf{v}^{12}, \mathbf{v}^{14}, \mathbf{v}^{15}$ & $\left(P_{2} ; P_{3} ; P_{5}\right)>0$ & $P_{8}=1$ \\
\hline 6 & $\mathbf{v}^{0}, \mathbf{v}^{4}, \mathbf{v}^{12}, \mathbf{v}^{13}, \mathbf{v}^{15}$ & $\left(P_{2} ; P_{4}\right)>0 ; P_{5} \leq 0$ & $P_{11}=1$ \\
\hline 7 & $\mathbf{v}^{0}, \mathbf{v}^{4}, \mathbf{v}^{5}, \mathbf{v}^{13}, \mathbf{v}^{15}$ & $P_{6}>0 ;\left(P_{3} ; P_{4}\right) \leq 0$ & $P_{11}=1$ \\
\hline 8 & $\mathbf{v}^{0}, \mathbf{v}^{1}, \mathbf{v}^{5}, \mathbf{v}^{13}, \mathbf{v}^{15}$ & $\left(P_{2} ; P_{3}\right)>0 ; P_{6} \leq 0$ & $P_{16}=1$ \\
\hline 9 & $\mathbf{v}^{0}, \mathbf{v}^{4}, \mathbf{v}^{6}, \mathbf{v}^{14}, \mathbf{v}^{15}$ & $\left(P_{1} ; P_{4}\right)>0 ; P_{3} \leq 0$ & $P_{8}=1$ \\
\hline 10 & $\mathbf{v}^{0}, \mathbf{v}^{4}, \mathbf{v}^{6}, \mathbf{v}^{7}, \mathbf{v}^{15}$ & $\left(P_{1} ; P_{5}\right)>0 ; P_{4} \leq 0$ & $P_{12}=1$ \\
\hline 11 & $\mathbf{v}^{0}, \mathbf{v}^{4}, \mathbf{v}^{5}, \mathbf{v}^{7}, \mathbf{v}^{15}$ & $P_{6}>0 ;\left(P_{3} ; P_{5}\right) \leq 0$ & $P_{12}=1$ \\
\hline 12 & $\mathbf{v}^{0}, \mathbf{v}^{1}, \mathbf{v}^{5}, \mathbf{v}^{7}, \mathbf{v}^{15}$ & $P_{1}>0 ;\left(P_{3} ; P_{6}\right) \leq 0$ & $P_{17}=1$ \\
\hline 13 & $\mathbf{v}^{0}, \mathbf{v}^{2}, \mathbf{v}^{6}, \mathbf{v}^{14}, \mathbf{v}^{15}$ & $\left(P_{2} ; P_{4}\right)>0 ; P_{1} \leq 0$ & $P_{9}=1$ \\
\hline 14 & $\mathbf{v}^{0}, \mathbf{v}^{2}, \mathbf{v}^{6}, \mathbf{v}^{7}, \mathbf{v}^{15}$ & $P_{6}>0 ;\left(P_{1} ; P_{4}\right) \leq 0$ & $P_{13}=1$ \\
\hline 15 & $\mathbf{v}^{0}, \mathbf{v}^{2}, \mathbf{v}^{3}, \mathbf{v}^{7}, \mathbf{v}^{15}$ & $P_{5}>0 ;\left(P_{2} ; P_{6}\right) \leq 0$ & $P_{13}=1$ \\
\hline 16 & $\mathbf{v}^{0}, \mathbf{v}^{1}, \mathbf{v}^{3}, \mathbf{v}^{7}, \mathbf{v}^{15}$ & $P_{2}>0 ;\left(P_{1} ; P_{5}\right) \leq 0$ & $P_{17}=1$ \\
\hline 17 & $\mathbf{v}^{0}, \mathbf{v}^{2}, \mathbf{v}^{10}, \mathbf{v}^{14}, \mathbf{v}^{15}$ & $P_{6}>0 ;\left(P_{2} ; P_{3}\right) \leq 0$ & $P_{9}=1$ \\
\hline 18 & $\mathbf{v}^{0}, \mathbf{v}^{2}, \mathbf{v}^{10}, \mathbf{v}^{11}, \mathbf{v}^{15}$ & $P_{4}>0 ;\left(P_{3} ; P_{6}\right) \leq 0$ & $P_{14}=1$ \\
\hline 19 & $\mathbf{v}^{0}, \mathbf{v}^{2}, \mathbf{v}^{3}, \mathbf{v}^{11}, \mathbf{v}^{15}$ & $P_{5}>0 ;\left(P_{2} ; P_{4}\right) \leq 0$ & $P_{14}=1$ \\
\hline 20 & $\mathbf{v}^{0}, \mathbf{v}^{1}, \mathbf{v}^{3}, \mathbf{v}^{11}, \mathbf{v}^{15}$ & $\left(P_{2} ; P_{3} ; P_{5}\right) \leq 0$ & $P_{18}=1$ \\
\hline 21 & $\mathbf{v}^{0}, \mathbf{v}^{8}, \mathbf{v}^{10}, \mathbf{v}^{14}, \mathbf{v}^{15}$ & $\left(P_{3}, P_{6}\right)>0 ; P_{1} \leq 0$ & $P_{7}=1$ \\
\hline 22 & $\mathbf{v}^{0}, \mathbf{v}^{8}, \mathbf{v}^{10}, \mathbf{v}^{11}, \mathbf{v}^{15}$ & $P_{5}>0 ;\left(P_{3} ; P_{6}\right) \leq 0$ & $P_{15}=1$ \\
\hline 23 & $\mathbf{v}^{0}, \mathbf{v}^{8}, \mathbf{v}^{9}, \mathbf{v}^{11}, \mathbf{v}^{15}$ & $P_{4}>0 ;\left(P_{1} ; P_{5}\right) \leq 0$ & $P_{15}=1$ \\
\hline 24 & $\mathbf{v}^{0}, \mathbf{v}^{1}, \mathbf{v}^{9}, \mathbf{v}^{11}, \mathbf{v}^{15}$ & $\left(P_{1} ; P_{3} ; P_{4}\right) \leq 0$ & $P_{18}=1$ \\
\hline
\end{tabular}

$S_{24}: \mathbf{v}^{0}-\mathbf{v}^{1}-\mathbf{v}^{9}-\mathbf{v}^{11}-\mathbf{v}^{15}-\mathbf{v}^{11}-\mathbf{v}^{9}-\mathbf{v}^{1}-\mathbf{v}^{0}$
TABELA X. VETORES DE COMUTAÇÃO, CONDIÇÕES DE

SEPARAÇ̃̃O E CONDIÇÕES LIMITES DE CADA SETOR DOS INVERSORES TRIFÁSICOS A QUATRO FIOS COM QUATRO BRAÇOS 